\title{
Incorporating floating surface objects into a fully dispersive surface wave model
}

\author{
Mark D. Orzech ${ }^{1 *}$, Fengyan $\mathrm{Shi}^{2}$, Jayaram Veeramony ${ }^{1}$, Samuel Bateman ${ }^{3}$, \\ Joseph Calantoni $^{3}$, and James T. Kirby ${ }^{2}$ \\ ${ }^{1}$ NRL Code 7320, 1009 Balch Blvd, Stennis Space Center, MS 39529 USA \\ ${ }^{2}$ Center for Applied Coastal Research, University of Delaware, Newark DE 19716 USA \\ ${ }^{3}$ NRL Code 7434, 1005 Balch Blvd, Stennis Space Center, MS 39529 USA \\ *Corresponding author. Phone: 1-228-688-5974. Fax: 1-228-688-4759. \\ Email: mark.orzech@nrlssc.navy.mil
}

\begin{abstract}
The shock-capturing, non-hydrostatic, three-dimensional (3D) finite-volume model NHWAVE was originally developed to simulate wave propagation and landslide-generated tsunamis in finite water depth (Ma et al., 2012). The model is based on the incompressible Navier-Stokes equations, in which the z-axis is transformed to a $\sigma$-coordinate that tracks the bed and surface. As part of an ongoing effort to simulate waves in polar marginal ice zones (MIZs), the model has now been adapted to allow objects of arbitrary shape and roughness to float on or near its water surface. The shape of the underside of each floating object is mapped onto an upper $\sigma$-level slightly below the surface. In areas without floating objects, this $\sigma$-level continues to track the surface and bed as before. Along the sides of each floating object, an immersed boundary method is used to interpolate the effects of the object onto the neighboring fluid volume. Provided with the object's shape, location, and velocity over time, NHWAVE determines the fluid fluxes and pressure variations from the corresponding accelerations at neighboring cell boundaries. The system was validated by comparison with analytical solutions and a VOF model for a 2D floating box and with laboratory measurements of wave generation by a vertically oscillating sphere. A steep wave simulation illustrated the high efficiency of NHWAVE relative to a VOF model. In a more realistic MIZ simulation, the adapted model produced
\end{abstract}

Preprint submitted to Ocean Modelling

April 7, 2016 
qualitatively reasonable results for wave attenuation, diffraction, and scattering.

Keywords: wave model, floating surface objects, sigma-coordinate, Navier-Stokes, finite volume, immersed boundary

\section{INTRODUCTION}

4

With the growing focus on global warming and its effects in the polar regions, increasing attention has been paid to modeling the interaction of waves with ice floes in marginal ice zones (MIZs) along the edges of Arctic and Antarctic pack ice. As areas of open water continue to grow in the Arctic during spring and summer months, more powerful waves are playing an increasingly important role in the breakup and recession of the sea ice (Thomson and Rogers, 2014). However, at present their representation in operational models such as WAVEWATCH III (Tolman, 2009) and Arctic Cap (Posey et al., 2010) is either crudely parameterized or completely neglected (Zhao et al., 2015). Development and validation of more accurate, physically based representations of wave-ice interaction is urgently needed.

Although mathematical representations of wave-ice interaction were first developed in the mid-20th century (Keller and Weitz, 1953; Evans and Davies, 1968; Wadhams, 1973; Squire et al., 1995), the sophistication and variety of theoretical and numerical wave-ice models accelerated considerably as polar research became a priority and more sophisticated semi-analytical models were developed (Meylan and Squire, 1994; Shen and Squire, 1998; Peter and Meylan, 2004; Bennetts et al., 2007; Kohout and Meylan, 2007). While some modelers have focused on representing the MIZ as a continuous material with varying viscoelastic properties (Zhao and Shen, 2013; Rogers and Orzech, 2013; Zhao and Shen, 2015), other investigators, beginning with Wadhams (Wadhams, 1986) and Meylan (Meylan, 1993; Meylan and Squire, 1996) have instead modeled individual floes as flexible thin elastic plates (Hermans, 2004; Andrianov and Hermans, 2004; Gayen et al., 2005). A common approach has been to use linear potential flow theory (as described in Squire, 2007) in combination with a thin- 
plate ice model to simulate wave-floe interaction (Kohout and Meylan, 2008; Williams et al., 2013; Meylan et al., 1997; Bennetts et al., 2010). While this method is very useful for estimating attenuation of smaller amplitude waves by floes, it neglects floe collisions and rafting, as well as the overwash of floes by steeper waves (Yiew et al., 2016). Results from several recent lab experiments (Toffoli et al., 2015; Bennetts et al., 2015; Bennetts and Williams, 2015) suggest that floe overwash plays a role in producing increased attenuation rates measured for steeper waves. Field measurements from Kohout et al. (2014) and Meylan et al. (2014) also indicate a possible amplitude dependence in wave transmission rates, which is not captured by linear potential theory or represented in larger scale ocean models. Bennetts and Williams (2015) note that floe collisions may reduce wave-energy transmission in more densely packed marginal ice fields. To address some of the issues associated with ice floe properties and behavior, other modelers have used the discrete element method (DEM) to directly represent single or multiple ice floes, simulating their evolution and tracking their motion in response to currents and/or waves (Hopkins and Thorndike, 2006; Hopkins and Shen, 2001; Hermans, 2013; Polojärvi and Tuhkuri, 2013; Xu et al., 2012; Song et al., 2014).

The wave model adaptations to be described herein are part of a larger effort to investigate and address the above issues by implementing a realistic 3D representation of the interactions between waves and Arctic ice floes in small domains of $\mathrm{O}\left(1-10 \mathrm{~km}^{2}\right)$. In this coupled system, waves are to be represented using the phase-resolving, fully dispersive surface wave model NHWAVE (Ma et al., 2012). Ice floes are represented using the open-source DEM package LIGGGHTS (Kloss et al., 2012), in a manner similar to the latter category of modelers described above (Orzech, et al., 2016; Bateman, et al., 2014; Orzech, et al., 2014). The system is limited to first-year ice, for which vertical thickness $(\mathrm{O}(m))$ is small relative to horizontal dimensions $(\mathrm{O}(100 m))$. This property makes it convenient to represent ice floe effects on waves as surface boundary conditions in the wave model.

The present article focuses on the wave component of this system, describing 
the development of numerical methods for the NHWAVE model to correctly represent the fluid response to rigid objects at its surface. Unlike most of the wave-ice models cited above, in which the motion of the freely floating floes is coupled to the waves, objects considered here are either fixed or moving with a prescribed oscillatory motion. As such, they are generally uncoupled from surrounding fluid oscillations and thus act to alter and/or generate surface waves in their vicinity. Vertically oriented surface object effects are incorporated into the model by directly mapping a near-surface $\sigma$-level onto the underside (and, if necessary, the top) of each floating object. Horizontally oriented effects are interpolated to fluid cell walls using extensions of the immersed boundary approach. The model is compared to potential-flow models for linear waves and 2D geometries, and to a lab experiment for a 3D geometry. Additional qualitative evaluations are performed for steep waves and a larger scale fieldbased case.

The following sections detail the specific changes made to NHWAVE, then describe and provide results from convergence tests, comparative analytical simulations, an experimental validation, and the qualitative steep-wave and largescale cases. Section 2 presents a comparative overview of the wave model in a historical context, followed by a brief review of the governing equations and numerical methods of NHWAVE. A more detailed description of the theory and methods for incorporating floating objects and mapping $\sigma$-levels is provided in Section 3. Validation test parameters and all test results are summarized in Section 4, and discussion and conclusions are presented in Section 5.

\section{WAVE MODEL}

\subsection{Background}

The behavior of floating objects and their interaction with water waves and currents have been studied from the earliest days of sailing and shipping. Theoretical and modeling efforts have primarily focused on how waves affect ships or structures, as in studies of nonlinear ship dynamics (Spyrou and Thomp- 
son, 2000), conditions leading to capsize (Soliman and Thompson, 1991; McCue and Troesch, 2005), and wave-object or wave-platform interactions (Wall et al., 2007; Clauss, 2002; Isaacson and Nwogu, 1987). Wave-structure interaction is usually modeled by the boundary integral method (BIM; e.g., Skourup et al., 1992; Grilli et al., 1994) or a computational fluid dynamics model (CFD; e.g., Hieu and Tanamoto, 2006; Shen and Chan, 2008). BIM is based on potential flow theory and does not allow for calculation of energy dissipation due to wave breaking and friction between flows and structures. Traditional CFD models use either the Volume-of-Fluid (VOF) method or the Marker-and-Cell (MAC) method to treat the free surface or the wave-structure interface and are thus computationally expensive. The smoothed particle hydrodynamics (SPH) method (Gingold and Monaghan, 1977), which treats the fluid as a collection of discrete elements, has also been used to model wave-object interaction (e.g. Rogers et al., 2010), but it is even more computationally demanding.

Models based on the multi-layer Boussinesq equations have been shown to be robust and efficient at simulation of non-linear wave propagation (Nwogu, 1993; Wei et al., 1995) and extended to represent turbulence in the water column (Kim and Lynett, 2011). However, assumptions inherent to these models (i.e., $k h<3)$ limit them to predicting only weakly dispersive shallow water waves in intermediate water depth. In addition, floating objects and the free surface itself can cut through their computational cells in an arbitrary way, significantly affecting the accuracy of their boundary conditions and ultimately degrading their velocity estimates.

The recently developed model NHWAVE (Ma et al., 2012) uses a different approach, solving the Navier-Stokes equations in a transformed domain with a surface/bed-following $\sigma$-coordinate instead of a Cartesian z-coordinate. The free surface is treated as a single-valued function of horizontal location, which resolves the issues with arbitrary intersection of cells discussed above. The model defines dynamic pressure at vertically-facing cell sides (i.e., along the $\sigma$-levels), which allows for accurate application of the pressure boundary condition at the free surface. With this model configuration, a very small number (i.e., fewer 
than 10) of vertical levels is required to accurately describe wave dispersion. The Harten Lax and van Leer (HLL) Riemann approximation (Harten et al., 1983 ) is used to determine fluxes at cell faces. A shock-capturing Godunovtype approach is employed, allowing the model to deal with discontinuous flow conditions resulting from breaking waves or sudden surface impacts. These simplifications also considerably reduce the computational requirements of the model.

The Pressure Decimation and Interpolation (PDI) Method was added to NHWAVE by Shi et al. (2015), who confirmed that the dynamic pressure can be modeled accurately with only a small number of vertical layers. This significantly increased model efficiency for simulating non-hydrostatic, baroclinic processes. Most recently, Derakhti et al. (2016b,c) carried out extensive model validations of NHWAVE against laboratory data. The focus of their study was to examine the model's capability of predicting breaking waves in both the surf zone and deep water. Results showed that NHWAVE accurately predicted depth-limited breaking waves using only $4 \sigma$-layers and steepness-limited breaking waves using as few as $8 \sigma$-layers.

\subsection{Governing Equations}

The complete NHWAVE equations can be found in Ma et al. (2012, 2013a,b). Here, we only list the equations related to the present implementation. Following the notation of Ma et al. (2012), the incompressible Navier-Stokes equations in Cartesian coordinates are given by

$$
\frac{\partial u_{i}}{\partial x_{i}}=0
$$

$$
\frac{\partial u_{i}}{\partial t}+u_{j} \frac{\partial u_{i}}{\partial x_{j}}=-\frac{1}{\rho} \frac{\partial P}{\partial x_{i}}+g_{i}+\frac{\partial \tau_{i j}}{\partial x_{j}}
$$

where $i$ and $j$ equal 1,2 , and 3 , with $\left(x_{1}, x_{2}, x_{3}\right)=(x, y, z), P$ equal to total pressure, $\rho$ the water density, $g$ the gravitational acceleration, $\tau_{i j}$ the turbulent shear stress, and $u_{i}$ the velocity component in the $x_{i}$ direction. The $z$ coordinate points up with an origin at the still water surface. With the transformation

$$
\sigma=(z+h) /(\eta+h)
$$


in which $h(x, y)$ is water depth and $\eta(x, y)$ is free surface elevation relative to still water level, (1) and (2) can be written in a compact, conservative form in the $\sigma$-coordinate (Shi et al., 2015):

$$
\frac{\partial \boldsymbol{\Psi}}{\partial t}+\nabla \cdot \boldsymbol{\Theta}(\boldsymbol{\Psi})=\mathbf{S},
$$

${ }_{148}$ where $\nabla=\left(\frac{\partial}{\partial x}, \frac{\partial}{\partial y}, \frac{\partial}{\partial \sigma}\right)$. Once it is discretized, (3) defines the $\sigma$-levels, which run from $\sigma=1$ at the free surface ("first" $\sigma$-level) to $\sigma=0$ at the seabed 150 ("last" $\sigma$-level). $\boldsymbol{\Psi}$ and $\boldsymbol{\Theta}(\boldsymbol{\Psi})$ are the vector of conserved variables and the 151 flux vector, respectively.

$$
\begin{gathered}
\boldsymbol{\Psi}=\left(\begin{array}{c}
D \\
D u \\
D v \\
D w
\end{array}\right) \\
\boldsymbol{\Theta}=\left(\begin{array}{c}
\left(D u u+\left(\frac{1}{2} g \eta^{2}+g h \eta\right)\right) \mathbf{i}+D u v \mathbf{j}+u \omega \mathbf{k} \\
D u v \mathbf{i}+\left(D v v+\left(\frac{1}{2} g \eta^{2}+g h \eta\right)\right) \mathbf{j}+v \omega \mathbf{k} \\
D u w \mathbf{i}+D v w \mathbf{j}+w \omega \mathbf{k}
\end{array}\right) .
\end{gathered}
$$

where $D=h+\eta,(u, v, w)$ represent velocity components in the Cartesian coordinate system $\left(x^{*}, y^{*}, z^{*}\right)$, and $\omega$ is defined as the vertical velocity in the $\sigma$ image domain (the relationship between $\omega$ and $u_{3}$ can be found in (Ma et al., 2012)). The source term on the right hand side includes several source components,

$$
\mathbf{S}=\mathbf{S}_{h}+\mathbf{S}_{p}+\mathbf{S}_{\tau}
$$


where $\mathbf{S}_{h}, \mathbf{S}_{p}$, and $\mathbf{S}_{\tau}$ represent the bottom slope term, dynamic pressure gradient, and turbulent mixing, respectively. These terms are expressed as

$$
\begin{array}{r}
\mathbf{S}_{h}=\left(\begin{array}{c}
g \eta \frac{\partial h}{\partial x} \\
g \eta \frac{\partial h}{\partial y} \\
0
\end{array}\right) \quad \mathbf{S}_{p}=\left(\begin{array}{c}
-\frac{D}{\rho}\left(\frac{\partial p}{\partial x}+\frac{\partial p}{\partial \sigma} \frac{\partial \sigma}{\partial x^{*}}\right) \\
-\frac{D}{\rho}\left(\frac{\partial p}{\partial y}+\frac{\partial p}{\partial \sigma} \frac{\partial \sigma}{\partial y^{*}}\right) \\
-\frac{1}{\rho} \frac{\partial p}{\partial \sigma}
\end{array}\right) \\
\mathbf{S}_{\tau}=\left(\begin{array}{c}
D S_{\tau x} \\
D S_{\tau y} \\
D S_{\tau z}
\end{array}\right),
\end{array}
$$

where $p$ represents the dynamic pressure. The $k-\epsilon$ closure model and the standard Smagorinsky (1963) Large Eddy Simulation (LES) model are implemented in NHWAVE (Derakhti et al. (2016a); note that these features are not used in the tests described below, for which objects are treated as frictionless). $\partial \sigma / \partial x^{*}$ and $\partial \sigma / \partial y^{*}$ are calculated by

$$
\begin{aligned}
& \frac{\partial \sigma}{\partial x^{*}}=\frac{1}{D} \frac{\partial h}{\partial x}-\frac{\sigma}{D} \frac{\partial D}{\partial x} \\
& \frac{\partial \sigma}{\partial y^{*}}=\frac{1}{D} \frac{\partial h}{\partial y}-\frac{\sigma}{D} \frac{\partial D}{\partial y}
\end{aligned}
$$

The governing equation for the free surface can be obtained by integrating the mass conservation equation over the full range of $\sigma$ (Ma et al., 2015) and is written as

$$
\frac{\partial D}{\partial t}+\frac{\partial}{\partial x}\left(D \int_{0}^{1} u d \sigma\right)+\frac{\partial}{\partial y}\left(D \int_{0}^{1} v d \sigma\right)=0
$$

\subsection{Numerical Implementation}

Numerically, NHWAVE uses a combined finite-volume and finite-difference scheme with a Godunov-type method for the spatial discretization and a twostage (second-order) SSP Runge-Kutta (R-K) scheme (Gottlieb et al., 2001) for time stepping. The two-step projection method, which splits the time integration into a hydrostatic predictor phase and the non-hydrostatic corrector phase, is used within each R-K stage. The computational grid is Cartesian in the horizontal direction and follows the $\sigma$-coordinate in the vertical direction. Here, we illustrate just the first stage of R-K for brevity. 
In the hydrostatic phase, an intermediate quantity, $\mathbf{U}=(D u, D v, D w)^{T}$, is evaluated using the momentum equations with the dynamic pressure term neglected,

$$
\frac{\mathbf{U}^{*}-\mathbf{U}^{n}}{\Delta t}=-\nabla \cdot \boldsymbol{\Theta}(\mathbf{U})^{n}+\mathbf{S}_{h}^{n}+\mathbf{S}_{\rho}^{n}+\mathbf{S}_{\tau}^{n},
$$

where $\mathbf{U}^{n}$ represents $\mathbf{U}$ value at time level $n$ and $\mathbf{U}^{*}$ is the intermediate value from the hydrostatic phase. The numerical fluxes $\Theta(\mathbf{U})$ in (11) are discretized using a second-order Godunov-type finite volume method.

In the non-hydrostatic phase, the velocity field is corrected using the dynamic pressure term:

$$
\frac{\mathbf{U}^{(1)}-U^{*}}{\Delta t}=\mathbf{S}_{p}^{(1)}
$$

where the superscript ()$^{(1)}$ represents the first stage for the R-K scheme. Substituting (12) into the continuity equation,

$$
\frac{\partial u}{\partial x}+\frac{\partial u}{\partial \sigma} \frac{\partial \sigma}{\partial x^{*}}+\frac{\partial v}{\partial y}+\frac{\partial v}{\partial \sigma} \frac{\partial \sigma}{\partial y^{*}}+\frac{1}{D} \frac{\partial w}{\partial \sigma}=0
$$

yields the Poisson equation of the dynamic pressure,

$$
\begin{aligned}
& \frac{\partial}{\partial x}\left[\frac{\partial p}{\partial x}+\frac{\partial p}{\partial \sigma} \frac{\partial \sigma}{\partial x^{*}}\right]+\frac{\partial}{\partial y}\left[\frac{\partial p}{\partial y}+\frac{\partial p}{\partial \sigma} \frac{\partial \sigma}{\partial y^{*}}\right]+\frac{\partial}{\partial \sigma}\left(\frac{\partial p}{\partial x}\right) \frac{\partial \sigma}{\partial x^{*}}+ \\
& \frac{\partial}{\partial \sigma}\left(\frac{\partial p}{\partial y}\right) \frac{\partial \sigma}{\partial y^{*}}+\left[\left(\frac{\partial \sigma}{\partial x^{*}}\right)^{2}+\left(\frac{\partial \sigma}{\partial y^{*}}\right)^{2}+\frac{1}{D^{2}}\right] \frac{\partial}{\partial \sigma}\left(\frac{\partial p}{\partial \sigma}\right)= \\
& \frac{\rho}{\Delta t}\left(\frac{\partial u^{*}}{\partial x}+\frac{\partial u^{*}}{\partial \sigma} \frac{\partial \sigma}{\partial x^{*}}+\frac{\partial v^{*}}{\partial y}+\frac{\partial v^{*}}{\partial \sigma} \frac{\partial \sigma}{\partial y^{*}}+\frac{1}{D} \frac{\partial w^{*}}{\partial \sigma}\right)
\end{aligned}
$$

The same procedure is used for the second stage of the R-K scheme.

The velocity and tracer variables are defined at the cell centers. The dynamic pressure for the Keller-box scheme is defined at the cell faces (Stelling and Zijlema, 2003). The velocity values at the cell faces, $\left(u^{*}, v^{*}, w^{*}\right)$, are obtained by linear interpolation using the adjacent cell-centered values for the discretization of the Poisson equation (14).

To model moving boundaries such as shorelines, NHWAVE includes a 2D moving mask scheme for alternately wet and dry cells. At each time step, the water depth is determined for each cell, and the cell is assigned a mask value of one (wet) if the depth is greater than a specified $D_{\min }$ and zero (dry) when 
depth is less than $D_{m i n}$. Dry cells have zero normal flux at cell faces, and wave speeds in neighboring wet cells are modified (see Ma et al., 2012).

\section{INCLUSION OF FLOATING OBJECTS}

To accomodate floating objects at the water surface, NHWAVE is extended to allow for modification of cell dimensions based on the characteristics of the object boundaries as well as those of the surface and bed (George, 2010). For a partially submerged floating object, the free surface (i.e., first $\sigma$-level) is broken at the object's lateral (or top) boundaries. (3) cannot be applied to surface regions occupied by an object. In these regions, NHWAVE is adapted to track the object's vertical motion by mapping a subsurface $\sigma$-level directly onto the underside of the object. The model's existing 2D moving-mask capability is upgraded to $3 \mathrm{D}$ to represent the interruption of the free surface by fully threedimensional objects. Kinematic and dynamic boundary conditions at lateral walls of cells touched by an object are determined using the immersed boundary (IB) method (Mittal and Iaccarino, 2005). Critical flow velocities are generally not an issue, as relevant applications involve slow-moving objects floating at (or near) the water surface. Wave breaking is included when warranted by more energetic conditions.

\subsection{Coordinate transformation}

In the vertical direction, a flexible $\sigma$-coordinate transformation is made for the given $z$ coordinates of the top and bottom of the object, $z_{t}$ and $z_{b}$, respectively (Figure 1). The $\sigma$-layers are divided into three regions, with constant sublayer thicknesses, $\Delta z$, for any horizontal location in each region specified as

$$
\begin{array}{cc}
\Delta z_{b}=\frac{h+z_{b}}{n_{b}} & \sigma=0 \sim k_{b} \\
\Delta z_{o}=\frac{z_{t}-z_{b}}{n_{o}} & \sigma=k_{b} \sim k_{t}
\end{array}
$$




$$
\Delta z_{t}=\frac{\eta-z_{t}}{n_{t}} \quad \sigma=k_{t} \sim 1,
$$

where subscripts $b, o$, and $t$ designate layers below, inclusive of, and above the object, respectively. $k_{b}$ and $k_{t}$ are $\sigma$-values of the bottom and top surfaces of the object, and $n_{b}, n_{o}$ and $n_{t}$ represent the number of layers in each region. For ice-floe applications, objects were restricted to the second layer from the top, with $n_{t}=1, n_{o}=1$.

In contrast to the free-surface form expressed by $(9), \frac{\partial \sigma}{\partial x}$ and $\frac{\partial \sigma}{\partial y}$ are calculated using a more general form as

$$
\begin{gathered}
\frac{\partial \sigma}{\partial x}=-\frac{\left.\frac{\partial z}{\partial x}\right|_{\sigma}}{\left.\frac{\partial z}{\partial \sigma}\right|_{x}} \\
\frac{\partial \sigma}{\partial y}=-\frac{\left.\frac{\partial z}{\partial y}\right|_{\sigma}}{\left.\frac{\partial z}{\partial \sigma}\right|_{y}}
\end{gathered}
$$

\subsection{D moving masks}

The existing code of NHWAVE uses the wetting-drying algorithm to simulate moving boundary processes. The wetting-drying method is implemented using a two-dimensional (2D) horizontal mask (e.g. Shi et al., 2012). The 2D mask is not applicable to modeling wave interactions with ice floes located at the water surface where waves and ice floes interact in three dimensions and waves diffract as they pass under floes. Therefore, the extension of the $2 \mathrm{D}$ mask to three dimensions (3D) was necessary.

The implementation was straightforward, following the existing 2D mask approach but taking into account the 3D geometry of individual objects. The mask array was initialized in three dimensions as $\operatorname{MASK}(i, j, k)$ with values of 0 or 1 assigned to cells containing object or fluid, respectively. A 3D object with geometry described in Cartesian coordinates $(x, y, z)$ was first projected onto the NHWAVE horizontal grid, $(x, y)$ as shown in Figure 2, after which the top and bottom surfaces of the object were fitted by the $\sigma$-coordinate as described 
in (15) - (17). Specifically, the mask values are assigned by

$$
\begin{array}{ll}
\text { MASK }=0 & \text { if object projection includes } u, v \text { point }(i, j) \text { and } k=k_{b} \sim k_{t} \\
\text { MASK }=1 & \text { otherwise. }
\end{array}
$$

The influence of objects moving in partially filled cells was taken into account with an immersed boundary method. The fluxes and accelerations (pressure effects) of an object were mapped to "boundary points" defined in fluid-filled cells immediately neighboring the partially filled cells marked by circled crosses in Figure 2. The boundary points were then used to apply the immersed boundary conditions. The implementation of this method in the kinematic and dynamic lateral boundary conditions is described in the following two sections, along with the simplified vertical boundary conditions resulting from the mapping of $\sigma$-levels.

\subsection{Dynamic boundary conditions}

In accordance with the pressure Poisson equation (14), the Neumann boundary condition is used for the dynamic pressure along object boundaries. For an object at the top or bottom of a cell,

$$
\frac{\partial p}{\partial \sigma}=-\rho D \frac{d w_{o}}{d t}
$$

where $\frac{d w_{o}}{d t}$ is the acceleration of the object in the vertical direction. Pressure components associated with the cell boundary conditions along mapped $\sigma$-layer boundaries are obtained using a central difference in the $\sigma$ direction. As illustrated in Figure 3, the central difference can be expressed by

$$
\frac{p_{i, j, k+1}-p_{i, j, k-1}}{2 \Delta \sigma}=-\rho_{0} D \frac{d w_{o}}{d t}
$$

In the horizontal direction, an object may move within a partially filled fluid cell. Under such conditions, we use the the direct-forcing immersed boundary method following Ma et al. (2015). An additional virtual forcing term, $S_{I B}$, was added to the non-hydrostatic phase,

$$
\frac{\mathbf{U}^{(1)}-U^{*}}{\Delta t}=\mathbf{S}_{p}^{(1)}+S_{I B}^{(1)},
$$


where $S_{I B}$ is defined as a Dirac delta function, which has non-zero values only at the boundary points defined in Figure 2. ()$^{(1)}$ is the first stage of the R-K scheme. The second stage should be performed in the same way. The virtual forcing at the boundary points was given by

$$
S_{I B}^{(1)}=\frac{\mathbf{u}_{b}-U^{*}}{\Delta t}-\mathbf{S}_{p}^{(1)}
$$

where $\mathbf{u}_{b}=\left(u_{b}, v_{b}\right)$, the velocity at a boundary point. The boundary velocity, $\mathbf{u}_{b}$, can be evaluated by linear interpolation or extrapolation using the fluid velocity at neighboring fluid points and the velocity of the object at the so-called marker points, as shown in Figure 3. For example, in the case demonstrated in Figure $3, u_{b}$ is obtained using the fluid velocity $u_{i-2, j, k}$ and the object velocity $u_{o}$ (marked by red arrow). Detailed model implementation and validation for the immersed boundary method can be found in Ma et al. (2015).

\subsection{Kinematic boundary conditions}

In the vertical direction, because the top and the bottom of the object are fitted by the $\sigma$-coordinate at every time step, the vertical velocity component $w\left(\right.$ or $\left.u_{3}\right)$ can be imposed directly through the kinematic boundary condition

$$
\left.w\right|_{z_{o}}=-\frac{\partial z_{o}}{\partial t}-\left.u\right|_{z_{o}} \frac{\partial z_{o}}{\partial x}-\left.v\right|_{z_{o}} \frac{\partial z_{o}}{\partial y}
$$

where $z_{o}$ is the $z$ coordinate of the object surface. (25) is of the same form as the kinematic boundary conditions used at the free surface and the bottom for bottom deformation problems (Ma et al., 2012). In addition, the vertical velocity $\omega$ in the image domain satisfies

$$
\omega=0 \text {. }
$$

Note that (26) is derived from the $\sigma$-coordinate transformation at the top and bottom surfaces of the object.

In the horizontal direction, as an object shifts within a partially filled fluid cell, its surface creates fluxes into or out of the cell. The numerical fluxes at 
the interface of a boundary cell, $\mathbf{u}_{s}$, can again be obtained by a linear interpolation/extrapolation method using the fluid velocity and the object velocity $\mathbf{u}_{o}$, as shown in Figure 3. Using (5) and (6), the kinematic boundary conditions can be expressed by

$$
\begin{gathered}
D u=D u_{s} \\
\Theta(D u)=D u_{s} u_{s}+\frac{1}{2} g \eta^{2}+g h \eta
\end{gathered}
$$

in the $x$ direction and

$$
D v=D v_{s}
$$

$$
\boldsymbol{\Theta}(D v)=D v_{s} v_{s}+\frac{1}{2} g \eta^{2}+g h \eta
$$

in the $y$ direction.

\section{NUMERICAL TESTS AND VALIDATION}

\subsection{Convergence tests}

The test of model convergence with the number of vertical layers in the $\sigma$ coordinate has been carried out in Ma et al. (2012). The test not only gave a good indication of convergence associated with the vertical discretization, but also verified that the Keller-box scheme (Stelling and Zijlema, 2003) can model surface waves accurately with relative few vertical layers.

In this study, we conduct general tests of model convergence with both horizontal space and time discretization. Following a general approach to a convergence test for a finite-difference or finite volume model, we set up the model in a rectangular domain of $800 \mathrm{~m} \times 800 \mathrm{~m}$, with a constant water depth of $10 \mathrm{~m}$. The initial condition is given by a motionless Gaussian hump of water with its center located at the center of the domain $\left(x_{c}, y_{c}\right)$,

$$
\eta(x, y, t=0)=a_{0} \exp -\gamma\left[\left(x-x_{c}\right)^{2}+\left(y-y_{c}\right)^{2}\right] / L^{2}
$$

where $a_{0}$ is the initial height of the hump, $L=10^{4} \mathrm{~m}$ is a scale length, and $\gamma=0.5$ is the shape coefficient. 
To test the convergence with respect to the horizontal spacing, we adopt a sequence of different grid spacing, $d x \times n$ and $d y \times n$, where $d x=d y=0.25$ $\mathrm{m}$, and $n=1,2, \ldots, 8$. The time step is kept as a constant, i.e., $d t=0.0125 \mathrm{~s}$ (The Courant Numbers are less than 0.5 in all the cases). Figure 4 shows the convergence rate with the horizontal grid refinement that is demonstrated by the RMS differences of simulated surface displacements between cases $n$ and $n+1$ at $t=20 \mathrm{~s}$. It shows that the logarithmic RMS differences decrease linearly as grid spacing decreases. The averaged Cauchy convergence rate is 1.11, which is consistent with the Godunov-type HLL scheme used in the model (HLL-Contact implemented in NHWAVE is not used for this study owing to its numerical instability).

The convergence with respect to time discretization is examined in a similar manner by using a sequence of time steps from 0.0125 to $0.2 \mathrm{~s}$ and keeping a constant horizontal grid spacing $d x=2 \mathrm{~m}$. The convergence rate with time step refinement is shown in Figure 5. It can be seen that the convergence rate decreases with a decrease of time step. The averaged convergence rate is 1.25 , which is a little lower than expected in terms of the second-order SSP Runge-Kutta scheme used in the time discretization. The reason for the reduced convergence rate at a smaller time step is unknown.

\subsection{Wave reflection and transmission by fixed floating objects}

Reflection and transmission play an important role in reshaping waves that interact with floating objects. The model accuracy can be evaluated using wave reflection and transmission coefficients defined by $K_{r}=H_{r} / H_{i}$ and $K_{t}=$ $H_{t} / H_{i}$, where $H_{i}, H_{r}$ and $H_{t}$ are the incident, reflected and transmitted wave height, respectively. An analytical solution for surface wave scattering by a rectangular floating object is provided by Mei and Black (1969), who use linear potential flow theory based on the formulation and numerical method of Miles (1967) for the summation of an infinite series.

Here we considered a two-dimensional floating object configured as a rectangular shape with a length of $2 a$ and a draft of $d$, in water of depth $h$ (Figure 
6). To compare with the solution of Mei and Black (1969), NHWAVE was set up in a vertical 2D computational domain $1600 \mathrm{~m}$ long and $10 \mathrm{~m}$ deep, with the horizontal coordinate specified by $x=0-1600 \mathrm{~m}$. The rectangular object was centered at $x=800 \mathrm{~m}$ with a draft of $d=5 \mathrm{~m}$. Monochromatic waves were generated by a wavemaker (Lin and Liu, 1998) located at $x=400 \mathrm{~m}$. We adopted a small incident wave amplitude $A_{0}=0.05 \mathrm{~m}$ to allow for comparison of results to the linear wave solution from Mei and Black (1969).

To avoid wave reflection from domain boundaries, sponge layers with a thickness of $300 \mathrm{~m}$ were used on both sides of the domain. Two configurations of the rectangular object were tested, with $a / d=1$ and $a / d=3$. The numerical experiments were performed using a series of wave periods, $T=5,6,8,10,12$ and $15 \mathrm{~s}$, to examine the transmission rates in response to different $k h$ values. These resulted in wavelengths of $36-144 \mathrm{~m}$ and wave steepness values $k a=0.002-0.009$. The horizontal grid size was $1 \mathrm{~m}$ except for the case of $T=5 \mathrm{~s}$ where a grid size of $0.5 \mathrm{~m}$ was used in order to resolve the short waves. Five vertical layers were applied to all cases with the object specified in the two top layers.

Figure 7 shows, as an example, the surface elevation from the model with the configuration $a / d=3$ and $T=10 s$. The model predicted the transmission of progressive waves of constant amplitude past the object as shown on the right side of the figure $(x=800-1400 \mathrm{~m})$. On the left side of the object $(x=400-800$ $\mathrm{m})$, partial standing waves were generated by the superposition of the incident waves and partially reflected waves. Model output was compared to results from Mei and Black (1969) in Figure 8. The model predicted transmission rates decreasing with increasing $k h$ values, which agrees very well with the analytical solutions. The model accuracy in predicting wave transmission was evaluated using the normalized root-mean-square error (NRMSE) with respect to the theoretical solutions. The NRMSE formula is defined as

$$
\mathrm{N} R M S E=\sqrt{\frac{\sum_{i=1}^{N}\left(X_{i}-X_{i}^{\prime}\right)^{2}}{\sum_{i=1}^{N} X_{i}^{2}}},
$$


where $N$ is the number of data values. $X^{\prime}$ and $X$ represent the model result and theoretical solution, respectively. NRMSEs were $1.2 \%$ and $3.2 \%$ for $a / d=1$ and 3 , respectively.

\subsection{Wave radiation from a moving object}

Another important test for wave-object interaction is the radiation and scattering of water waves by a moving object. Theoretical solutions of wave radiation from a moving object, such as heaving, surging and pitching, have been derived by several authors. The solutions are based on linear wave theory and may be used for the present model tests given approximately linear wave conditions.

Analytical solutions of wave radiation from a heaving object can be found in Black et al. (1971), where Schwinger's variational formulation is applied to the radiation of surface waves due to small oscillations of bodies. In the numerical model tests, we set up the model in the same manner as in the wave refraction tests shown in Figure 6. To compare with Black et al. (1971), the rectangular object was specified with $d=5 \mathrm{~m}$ and $a=5 \mathrm{~m}$. The object oscillated with vertically sinusoidal motion and a small amplitude $(A=0.1 \mathrm{~m})$, with several different periods. Figure 9 shows the modeled wave amplitude Amp normalized by the oscillation amplitude $A$ in comparison to the analytical solutions. Model output was close to theory, with a slight underprediction of normalized amplitudes at some frequencies. The NRMSE defined by (32) was $8.3 \%$ for this case.

Wave radiation and scattering from a surging and pitching object were tested against analytical solutions from Zheng et al. (2004). The model setups were similar to the heaving test case except that the motions of the object were prescribed to be surging and pitching. For the surging case, a sinusoidal motion was specified in the $x$ direction with amplitude $A$ again equal to $0.1 \mathrm{~m}$ and a range of oscillation periods. Figure 10 shows the model results with comparisons to the analytical solutions, in the same manner as in Figure 9. The model predicted the wave amplitudes reasonably well with some underprediction for lower frequency motions. NRMSE was $5.9 \%$. Further tests with higher model 
resolution $(0.5 \mathrm{~m})$ reduced this error slightly to $5.6 \%$.

In the tests with a pitching object, the object motion was specified by the amount of pitch with respect to the centroid of the object, i.e., $x=0 \mathrm{~m}$ and $z=0 \mathrm{~m}$. In this case, a maximum pitch amplitude of $A=0.1 \mathrm{~m}$ was prescribed at the outer edge of the object (i.e., at $x=a$ ). Figure 11 shows the comparison between the model results and analytical solutions. The model predicted the peak of the wave height at the resonant frequency well and exhibited good agreement with the analytical solutions for all $k h$ values. NRMSE was $2.2 \%$ for this case.

The configuration of the heaving, surging, and pitching cases above is atypical for the adapted NHWAVE model, which is configured to represent surface objects that are thin relative to overall water depth, restricting those objects to the first two $\sigma$-layers near the surface. A block with a draft of $5 \mathrm{~m}$ in a depth of $10 \mathrm{~m}$ causes considerable distortion of the fitted upper $\sigma$-levels in the wave model. Despite these conditions, the output of the model in all three cases is reasonably close to theory, with an exceptionally small error in the pitching test.

\subsection{Steep waves passing a series of floating objects}

The above model tests used a small wave amplitude and floating objects with small aspect ratios in order to compare with theoretical solutions based on linear wave theory. In contrast, the main applications of the planned wave-ice system will be to scenarios where waves may be steep and the aspect ratio of typical first-year ice floes may be much larger than those in the above tests. We conducted an additional test in which steeper waves pass through a series of floating objects with a larger aspect ratio. Lacking experimental data and theoretical solutions for such a problem, the model results from NHWAVE are compared with a validated VOF-type Navier-Stokes solver (Shi et al., 2004). As mentioned in the introduction, a VOF-type model uses a function of a volume of fluid to capture the wave surface and the solid-fluid interface. A high grid resolution is needed for this application.

NHWAVE and the VOF model were set up in a $800 \mathrm{~m}$ long and $10 \mathrm{~m}$ deep 
computational domain. Four fixed floating objects with a width of $50 \mathrm{~m}$ and draft of $1 \mathrm{~m}$ were added at locations from $250 \mathrm{~m}$ to $750 \mathrm{~m}$ as shown in Figure 12. To make the wave generation exactly the same in the two models, waves were generated by specifying the theoretical solution at the left boundary instead of using the internal wavemaker (the internal wavemaker theories are different for the two models). Sponge layers were not applied. In NHWAVE, the horizontal grid size was $1 \mathrm{~m}$, and five $\sigma$ layers were used. In the VOF model, the horizontal grid size was also $1 \mathrm{~m}$, and a stretched grid was adopted in the vertical direction with the minimum grid size of $0.01 \mathrm{~m}$ around the still water level in order to capture the wave surface and the water-object interface. The total number of vertical layers was 120 .

Waves were generated based on second-order Stokes wave theory. The incident wave height was $1 \mathrm{~m}$ and the wave period was $8 \mathrm{~s}$, resulting in an incident steepness $k a \approx 0.09$. The waves did not overwash the floes. Figure 12 shows comparisons of surface elevation between the NHWAVE results (blue solid lines) and the VOF-model results (red dashed lines) at $t=20 \mathrm{~s}, 40 \mathrm{~s}, 60 \mathrm{~s}$ and $80 \mathrm{~s}$. Wave transmission and reflection processes are demonstrated in the snapshots of surface elevation from the two models, where the wave amplitudes and phases were very close to each other. Qualitatively, these results display the reflection and transmission characteristics typically seen for steep waves in ice floes; i.e., transmitted waves become progressively less steep as they pass successive ice floes. Note that the VOF-model uses a much higher vertical resolution than NHWAVE. While results are similar, the computational cost of the VOF model in this application is an order of magnitude higher than that of NHWAVE.

\subsection{Waves generated by an oscillating sphere}

Model output is further validated by comparison with the results of a 3D laboratory experiment. While there are many physical tests measuring the effects of waves on floating objects, relatively few examine the waves and other disturbances generated in a fluid by the motions of surface objects. The most common type of study that includes object-generated wave effects is the analysis of ship 
wakes (e.g., Soomere, 2007; Miyata et al., 1992; Torsvik et al., 2009; Soomere, 2005), where waves tend to be large in size and fluid velocities are relatively high. Most laboratory experiments examine the effects of bodies impacting the water at high speed, as seen in landslides (e.g., Panizzo et al., 2002; Heller et al., 2008; Di Risio et al., 2009). We were unable to find any three-dimensional studies in which waves were recorded in a reproducible manner from an object oscillating vertically at more moderate speeds. To obtain such a dataset, we found it necessary to design and implement our own lab experiment.

The experiment was conducted in a cylindrical water tank of $3 \mathrm{~m}$ diameter and $2 \mathrm{~m}$ depth (Figure 13), in which a $22 \mathrm{~cm}$ diameter solid sphere (bowling ball) was attached to a thin cable and manually oscillated up and down at the water surface for several seconds. A metal rod was bolted to the top of the sphere and passed through a vertical pipe on the mounting bracket to ensure consistent vertical motion. The positions of the sphere and the resulting waves were tracked along a horizontal axis with a Riegl VZ1000 scanning LIDAR system (Riegl, 2014). Small pieces of confetti paper were added to the water to enhance the LIDAR reception of surface shape. The LIDAR was operated in line scan mode with a scanning rate of $10 \mathrm{~Hz}$.

After allowing the water surface to become completely calm, the sphere was oscillated over a vertical range of about $\pm 5 \mathrm{~cm}$ at a frequency of roughly 2 $\mathrm{Hz}$ for approximately 10 seconds. The positions of the sphere and the water surface $( \pm 2-3 \mathrm{~mm})$ were recorded by the LIDAR. To avoid contamination by waves reflected from the tank wall, only data from the first $2.5 \mathrm{~s}$ were utilized. A higher resolution time series $(d t=0.01 s)$ of sphere position was required for the NHWAVE computation and was extracted from the $10 \mathrm{~Hz}$ LIDAR data by running a cubic spline interpolation. The vertical scale of the test was very small, with measured wave heights not exceeding $4 \mathrm{~cm}$.

To compare with the experimental results, NHWAVE was set up in a $3 \mathrm{~m}$ $\times 3 \mathrm{~m}$ rectangular domain with a configuration otherwise matching that in the tank experiment and set to record water level time series at several locations increasingly farther from the sphere. The grid size was $0.5 \mathrm{~m}$. The NHWAVE 
surface elevation time series are plotted against LIDAR elevation data at four locations in Figure 14.

Model performance was reasonably good, with wave amplitudes generally predicted well but with slight phase shifts in some wave peaks. The relative errors of the maximum wave height, calculated by $\left|H_{\text {data }}-H_{\text {model }}\right| / H_{\text {data }}$, where $H_{\text {data }}$ and $H_{\text {model }}$ represent the maximum envelope of the surface elevation, were $1.1 \%, 2.3 \%, 1.71 \%$ and $0.70 \%$, respectively, at the four measurement locations $(\mathrm{r}=0.45 \mathrm{~m} \sim 1.05 \mathrm{~m}$ ). Absolute errors in maximum wave height ranged from $0.2-0.5 \mathrm{~mm}$. Factors that may contribute to these errors include neglected surface and form drag, nonlinear behavior of the fluid in the immediate vicinity of the sphere, and errors in interpretation of the LIDAR elevation data for the sphere and the water surface.

Surface and form drag were both small for the smooth bowling ball, which oscillated over a less-than $10 \mathrm{~cm}$ range at under $2 \mathrm{~Hz}$. These conditions also limited the role of nonlinear effects resulting from flow separation and associated turbulence. The waves in the $2 \mathrm{~m}$ tank were deep water waves, with $k h \approx 32$. It is likely that measurements based on LIDAR - including surface elevation, horizontal locations, and possibly also time steps - are the greatest contributors to overall error in the experiment. Analysis of the LIDAR dataset revealed several millimeters of uncertainty in surface elevations, as described above. We were unable to determine the relative quality of individual elevation measurements, so the final elevation results were determined by a simple averaging of elevation values recorded within $\pm 5 \mathrm{~mm}$ (horizontally) of each selected location at each time step. This averaging of LIDAR horizontal and vertical data very likely contributed to both the amplitude and the phase differences between measured and modeled surface time series.

\subsection{A large domain simulation}

To examine the model's performance in simulating wave-ice floe interaction for a larger computational domain with more realistic floe size distribution, we set up a test in a $1.2 \mathrm{~km} \times 1 \mathrm{~km}$ domain with a constant water depth of $10 \mathrm{~m}$. 
The horizontal coordinates were configured as $x=0 \sim 1200 \mathrm{~m}$ and $y=0 \sim 1000$ m. Ice floes with different sizes and a uniform draft of $2 \mathrm{~m}$ were distributed at fixed locations throughout the domain (motion of the floes was not considered). The ice floe size distribution followed Toyota et al. (2006)'s field observation, i.e., the ice floe distribution $N$ was estimated empirically as a function of floe diameter $d$ as

$$
N(d)=N_{0} d^{-\alpha}
$$

where $N_{0}$ is a parameter representing the general density of ice floes and $\alpha$ is an empirical coefficient obtained by the least squares method using field data. The parameter $\alpha$ has a large range and depends on the size range of the ice floes. For floes smaller than $40 \mathrm{~m}$, a reasonable estimate is $\alpha=1.15$. In this case, we adopted $N_{0}=1500.0$ and $\alpha=1.15$. The floes were represented as square blocks, with side lengths discretized into 20, 25, 30, 35, and $40 \mathrm{~m}$. The total number of ice floes was 160 based on (33). The floes were distributed in the region $x=300 \sim 1100 \mathrm{~m}$ and $y=100 \sim 900 \mathrm{~m}$. A random function was used to assign each floe location.

The model grid size was $2 \mathrm{~m}$ in both $x$ and $y$ directions. Five vertical layers were used. The internal wavemaker was located at $x=100 \mathrm{~m}$. Normally incident waves were generated using the JONSWAP spectral distribution with a significant wave height of $1 \mathrm{~m}$ and peak period of $8 \mathrm{~s}$. Waves did not overwash the floes. Sponge layers of $50 \mathrm{~m}$ were used at the four lateral boundaries.

Figure 15 shows a snapshot of wave surface elevation and ice floes in the domain (wavemaker and sponge layers are not shown). Results are qualitatively reasonable. As waves pass through the irregularly distributed floes, stems are formed due to the combination of reflected and transmitted waves. Wave diffraction can be seen behind groups of ice floes and larger individual floes. Wave magnitude attenuates with increasing distance through the field of ice floes.

This test was performed on the Linux cluster Farber located at the University of Delaware. Using 48 cores, it took about 8 hours for a simulation of $1000 \mathrm{~s}$. The $10 \mathrm{~m}$ water depth was selected to reduce the computational time step (via 
${ }_{548}$ the CFL condition). With a depth of $100 \mathrm{~m}$, this simulation would require 549 roughly 24 hours. A more detailed analysis of wave scattering, transmission 550 rates and wave breaking, including validation with available laboratory and 551 field data, will be conducted when the fully coupled NHWAVE-DEM system 552 becomes operational. 


\section{DISCUSSION AND CONCLUSIONS}

As part of a larger effort to model wave interaction with Arctic ice floes, the non-hydrostatic, finite volume model NHWAVE has been modified to accomodate floating surface objects. A flexible coordinate transformation was added to the $\sigma$-coordinate (i.e., vertical) dimension of the model, allowing $\sigma$-layers in the vicinity of a floating object to be mapped directly onto the top and bottom surfaces of the object. The existing 2D horizontal mask for wetting and drying was extended to 3D to keep track of fluid cells that are partially or completely occupied by a floating object. Using an immersed boundary method, kinematic boundary conditions were modified to account for the fluid fluxes generated in neighboring cells by the motion of the fluid/object interface, and dynamic boundary conditions were added to calculate changes in fluid pressure due to vertical and horizontal accelerations of the floating object.

Model convergence rates for space and time discretization were verified to be within normal ranges. The adapted model was then validated by comparison to analytical solutions for wave transmission, reflection, and generation by a floating rectangular block. Model-estimated results compared well to theory for all tests, although generated wave heights were somewhat underestimated at lower oscillation frequencies for a heaving or surging block. The modest underestimations resulted from the relatively large draft of the floating object used, the oversimplified representation of the object motion in the theoretical derivation, and the smaller amplitude motions not fully resolved in virtual forcing computed by the immersed boundary method.

A qualitative comparison was provided for a case of steep waves passing through multiple ice floes. Results from NHWAVE closely matched those from a VOF solver, with both models predicting transmission of lower frequency wave components and reflection of higher frequency wave components, reducing the steepness of transmitted waves. The adapted model was also much more efficient, producing the output roughly ten times faster than the VOF model.

NHWAVE was next validated by comparison to measurements from a labora- 
tory experiment in which waves were created by a vertically oscillating sphere. Surface elevation time series of small amplitude $(\mathrm{O}(10 \mathrm{~cm}))$ waves were reasonably well matched by the model at four locations, with a slight difference in phase apparent at some locations. The phase difference was likely due primarily to LIDAR-related errors in measurement of surface elevations. A smaller contribution to model error came from surface and form drag effects that were not yet included in this version of NHWAVE. The implementation of these additional features represents future work.

For a final qualitative evaluation, the adapted model was used in a largerscale domain to simulate wave transmission, reflection, and diffraction by a field of ice floes with a realistic floe size distribution. Simulation results indicated significant reflection and scattering of incident waves by the floes, with evidence of diffraction behind larger ice pieces. Wave attenuation resulting from overwash of floes was not included in this case or the earlier steep wave case, but tests will be performed in the near future with the coupled wave-ice system to compare model output to recent lab results from Toffoli et al. (2015) and Bennetts et al. (2015).

Overall wave model performance was satisfactory in all validations of NHWAVE with floating objects present in the simulation domain. The modifications to NHWAVE presented here represent a key component to the larger wave-ice modeling project. Future work will be focused on coupling NHWAVE with the discrete element method to represent ice floes as collections of small bonded particles. When fully coupled, the two models will simulate the exchange of momentum and energy between waves and ice floes, including refraction, reflection, and diffraction of waves as well as losses due to shear, fracturing, overwash, and collision of floes. While this research is primarily oriented toward ice floes in waves, the adapted model described here could potentially be applied to other floating objects in deep water such as barges, ships, platforms, flotsam or garbage patches. 


\section{ACKNOWLEDGMENTS}

This work was supported under base funding to the Naval Research Laboratory from the Office of Naval Research. The work was also supported in part by a grant of computer time from the DoD High Performance Computing Modernization Program at the ERDC DSRC and Navy DSRC. FS received support from Office of Naval Research Award \#N0014-14-0569. We thank the technical support team from code 7434 for their invaluable assistance in completing the infamous "bowling ball experiment". We also thank the three anonymous reviewers for their detailed and constructive comments, which have significantly improved this manuscript.

\section{REFERENCES}

Andrianov, A.I., Hermans, A.J., 2004. Hydroelasticity of elastic circular plate. In: Landrini, M., Campana, E.F., Iafrati, A. (Eds.), Proc. 19th Intl. Workshop on Water Waves and Floating Bodies. Istituto Nazionale Studi ed Esperienze di Architettura Navale, Cortona, Italy.

Bateman, S., Shi, F., Orzech, M., Veeramony, J., Calantoni, J., 2014. Discrete element method simulations of ice floe dynamics. AGU 2014 Fall Meeting, Abstract EP43C-3580, San Francisco, CA.

Bennetts, L.G., Briggs, N.R.T., Porter, D., 2007. A multi-mode approximation to wave scattering by ice sheets of varying thickness. J. Fluid Mech. 579, 413-443.

Bennetts, L.G., Peter, M.A., Squire, V.A., Meylan, M.H., 2010. A threedimensional model of wave attenuation in the marginal ice zone. J. Geophys. Res. 115, C12043.

Bennetts, L.G., Alberello, A., Meylan, M.H., Cavaliere, C., Babanin, A.V., Toffoli, A., 2015. An idealised experimental model of ocean surface wave transmission by an ice floe. Oc. Mod. 96, 85-92. 
Bennetts, L.G., Williams, T.D., 2015. Water wave transmission by an array of floating discs. Proc. R. Soc. A 471, 20140698.

Black, J.L., Mei, C.C., Bray, M.C.G., 1971. Radiation and scattering of water waves by rigid bodies. J. Fluid Mech. 46, 151-164.

Derakhti, M., Kirby, J. T., Shi, F., and Ma, G., 2016a. NHWAVE: Governing equations, consistent boundary conditions and turbulence modeling. Oc. Mod., under review.

Derakhti, M., Kirby, J. T., Shi, F., and Ma, G., 2016b. Wave breaking in the surf zone and deep water in a non-hydrostatic model. Part 1: Cross-shore wave transformation. Oc. Mod., in revision.

Derakhti, M., Kirby, J. T., Shi, F., and Ma, G., 2016c. Wave breaking in the surf zone and deep water in a non-hydrostatic model. Part 2: Breaking-induced velocity and turbulence. Ocean Modelling, in revision.

Clauss, G. F., 2002. Dramas of the sea: episodic waves and their impact on offshore structures. Appl. Oc. Res 24, 147-161.

Di Risio, M., Bellotti, G., Panizzo, A., 2009. Three-dimensional experiments on landslide generated waves at a sloping coast. Coast. Engrg. 56(5-6), 659-671.

Doctors, L.J., 2003. The influence of viscosity on the wavemaking of a model catamaran. Proc. 18th Int'l Wkshp on Water Waves and Floating Bodies, Le Croisie, France 12, 1-4.

Eldhuset, K., 1996. An automatic ship and ship wake detection system for spaceborne SAR images in coastal regions. IEEE Trans. on Geosci. and Rem. Sens. 34(4), 1010-1019.

Evans, D.V., Davies, T.V., 1968. Wave-ice interaction. Rep. 1313, Davidson Lab., Stevens Inst. Technol., Hoboken, NJ.

Gayen, R., Mandal, B.N., Chakrabarti, A., 2005. Water-wave scattering by an ice-strip. J. Eng. Math. 53(1), 21-37. 
George, D.L., 2010. Adaptive finite volume methods with well-balanced Riemann solvers for modeling floods in rugged terrain: application to the Malpasset dam-break flood (France, 1959). Int. J. Numer. Meth. Fluids 66, 10001018.

Gingold, R.A., Monaghan, J.J., 1977. Smoothed particle hydrodynamics: theory and application to non-spherical stars. Mon. Not. R. Astron. Soc. 181, 375389.

Gottlieb S., Shu C.-W. and Tadmor E., 2001. Strong stability-preserving highorder time discretization methods. SIAM Review 43, 89-112.

Grilli, S.T., Losada, M.A. and Martin, F., 1994. Characteristics of solitary wave breaking induced by breakwaters. J. Water. Port Coastal \& Oc. Engrg. 120, $1,74-92$.

Harten, A., Lax, P., van Leer, B., 1983. On upstream differencing and Godunov type methods for hyperbolic conservation laws. SIAM Review 25(1), 35-61.

Heller, V., Hager, W. H., Minor, H.E., 2008. Scale effects in subaerial landslide generated impulse waves. Exp. in Fluids 44(5), 691-703.

Hermans, A., 2013. Interaction of free-surface waves with floating flexible strips. J. Eng. Math. 49(2), 133-147.

Hermans, A., 2013. Numerical modeling of force and contact networks in fragmented sea ice. Annals Glaciol. 54(62), 114-120.

Hieu, P.D. and Tanimoto, K., 2001. Verification of a VOF-based two-phase flow model for wave breaking and wave-structure interactions. Oc. Engrg. 33, 11-12 1565-1588.

Hopkins, M. A., Shen, H. H., 2001. Simulation of pancake ice in a wave field. Annals Glaciol. 33, 355-360.

Hopkins, M. A., Thorndike, A. S., 2006. Floe formation in Arctic sea ice. J. Geophys. Res. 111, C11S23. 
Isaacson, M., Nwogu, O. U., 1987. Wave loads and motions of long structures in directional seas. Trans. ASME 109, 126-132.

Keller, J.B., Weitz, M., 1953. Reflection and transmission coefficients for waves entering or leaving an icefield. Commun. Pure Appl. Math. 6(3), 415-417.

Kim, D. H., Lynett, P. J., 2011. Turbulent mixing and passive scalar transport in shallow flows. Phys. Fluids 23(1), 016603.

Kloss, C., Goniva, C., Hager, G., Amberger, S., Pirker, S., 2012. Models, algorithms and validation for opensource DEM and CFD-DEM. Progress in Comp. Fluid Dyn., An Int. J. 12(2/3), 140-152.

Kohout, A.L., Meylan, M.H., 2007. A model for wave scattering in the marginal ice zone based on a two-dimensional floating elastic plate solution. Ann. Glaciol. 44, 101-107.

Kohout, A.L., Meylan, M.H., 2008. An elastic plate model for wave attenuation and ice floe breaking in the marginal ice zone. J. Geophys. Res. 113(C9), C09016.

Kohout, A.L., Williams, M.J.M., Dean, S.M., Meylan, M.H., 2014. Storminduced sea-ice breakup and the implications for ice extent. Nature 509, 13262.

Lee, C.M., Cole, S., Doble, M., Freitag, L., Hwang, P., Jayne, S., Jeffries, M., Krishfield, R., Maksym, T., Maslowski, W., Owens, B., Posey, P., Rainville, L., Roberts, A., Shaw, W., Stanton, T., Thomson, J., Timmermans, M., Toole, J., Wadhams, P., Wilkinson, J., bibinfoauthorZhang, J., bibinfoyear2012. Marginal Ice Zone (MIZ) Program: Science and Experiment Plan. APL-UW TR1201, Technical Report, Applied Physics Laboratory, University of Washington, Seattle, 48pp..

Liang, Q., Marche, F., 2009. Numerical resolution of well-balanced shallow water equations with complex source terms. Adv. Water Res. 32, 873-884. 
Lin, P., Liu, P.L.F., 1998. Turbulence transport, vorticity dynamics, and solute mixing under plunging breaking waves in surf zone. J. Geophys. Res. 103(C8), $15,677-15,694$.

Ma, G., Shi, F., Kirby, J. T., 2012. Shock-capturing non-hydrostatic model for fully dispersive surface wave processes. Ocean Modelling 43-44, 22-35.

Ma, G., F. Shi, and J. T. Kirby, 2013a. Numerical simulation of tsunami waves generated by deformable submarine landslides, Oc. Mod., 69, 146-165.

Ma, G., J. T. Kirby, S. F. Su, J. Figlus and F. Shi, 2013b, Numerical study of turbulence and wave damping induced by vegetation canopies, Coastal Engineering, 80, 68-78.

Ma, G., A. A. Farahani and F. Shi, 2015, Modeling wave-structure interactions by an immersed boundary method in a $\sigma$-coordinate model, in preparation.

McCue, L., Troesch, A., 2005. Probabilistic determination of critical wave height for a multi-degree of freedom capsize model. Oc. Engrg. 32, 1608-1622.

Mei, C.C., Black, J.L., 1969. Scattering of surface waves by rectangular obstacles in waters of finite depth. J. Fluid Mech. 38, 499-511.

Meylan, M.H., 1993. The behaviour if sea ice in ocean waves. PhD Thesis, University of Otago, Dunedin, New Zealand.

Meylan, M.H., Squire, V.A., Fox, C., 1997. Towards realism in modeling ocean wave behavior in marginal ice zones. J. Geophys. Res. 102, 22981-22991.

Meylan, M.H., Bennetts, L.G., Kohout, A.L., 2014. In situ measurements and analysis of ocean waves in the Antarctic marginal ice zone. Geophys. Res. Lett. 41, 5046-5051.

Meylan, M.H., Squire, V.A., 1994. The response of ice floes to ocean waves. J. Geophys. Res. 99(C1), 899-900. 
Meylan, M.H., Squire, V.A., 1996. Response of a circular ice floe to ocean waves. J. Geophys. Res. 101(C4), 8869-8884.

Miles, J.W., 1967. Electrostatics of slender bodies. J. Appl. Phys. 38(1), 192196.

Mittal, R., Iaccarino, G., 2005. Immersed boundary methods. Annual Review of Fluid Mechanics 37, 239-261.

Miyata, H., Ming, Z., Watanabe, O., 1992. Numerical study on a viscous-flow with free-surface waves about a ship in steady straight course by a finite volume method. J. of Ship Res. 36(4), 332-345.

Nwogu, O., 1993. Alternative form of Boussinesq equations for nearshore wave propagation. J. Waterway, Port, Coastal and Ocean Engrg 119, 618-638.

Nwogu, O., 2007. Numerical modeling of waves generated by high-speed vessels in shallow water with a coupled Boussinesq-panel method. 9th Int'l Conf. on Num. Ship Hydrodyn., Ann Arbor, MI, Aug 5-8, 2007.

Orzech, M., Veeramony, J., Shi, F., Bateman, S., Calantoni, J., 2016. Modeling small-scale physics of waves and ice in the MIZ. AGU 2016 Ocean Sciences Meeting, Session 9483, Abstract 89970, New Orleans, LA.

Orzech, M., Shi, F., Calantoni, J., Bateman, S., Veeramony, J., 2014. Smallscale modeling of waves and floes in the marginal ice zone. AGU 2014 Fall Meeting, Abstract C11A-0347, San Francisco, CA.

Panizzo, A., Belotti, G., De Girolamo, P., 2002. Application of wavelet transform analysis to landslide generated waves. Coast. Engrg. 44(4), 321-338.

Peter, M.A., Meylan, M.H., 2004. Infinite-depth interaction theory for arbitrary floating bodies applied to wave forcing of ice floes. J. Fluid Mech. 500, 145167. 
Polojärvi, A., Tuhkuri, J., 2013. On modeling cohesive ridge keel punch through tests with a combined finite-discrete element method. Cold Reg. Sci. Tech. 85, 191-205.

Posey, P.G., Metzger, E.J., Wallcraft, A.J., Smedstad, O.M., Phelps, M.W., 2010. Validation of the 1/12 Arctic Cap Nowcast/Forecast System (ACNFS). NRL Report NRL/MR/7320-10-9287.

Riegl Laser Measurement Systems, 2014. Produktdetail, Riegl VZ-1000. http://www.riegl.com/nc/products/terrestrial-scanning/, visited Dec. 9, 2014.

Rogers,B., Dalrymple, R., Stansby, P., 2010. Simulation of caisson breakwater movement using 2-D SPH. J. Hydraul. Res. 48, 135-141.

Rogers, W.E., Orzech, M.D., 2013. Implementation and testing of ice and mud source functions in WAVEWATCH III. Naval Research Laboratory Memorandum Report 7320-13-9462, April 2013.

Shen, L. and Chan, E.S., 2008. Numerical simulation of fluid-structure interaction using a combined volume of fluid and immersed boundary method. Oc. Engrg. 35(8-9), 939-952.

Shen, H.H., Squire, V.A., 1998. Wave damping in compact pancake ice fields due to interactions between ice cakes. In: Jeffries, M. (Ed.), Antarctic Sci. Res. Series, Amer. Geophys. Union 74, 325-341.

Shi, F., Zhao, Q., Kirby, J. T., Lee, D.S., Seo, S.N., 2004. Modeling of wave interaction with complex coastal structures using an enhanced VOF model. Proc. 29th Conf. Coastal Engrg., Lisbon, Portugal, 13pp.

Shi, F., Kirby, J. T., Harris, J. C., Geiman, J. D., Grilli, S. T., 2012. A highorder adaptive time-stepping TVD solver for Boussinesq modeling of breaking waves and coastal inundation. Oc. Mod. 43-44, 36-51.

Shi, J., Shi, F.,Kirby, J. T., Ma, G., Wu, G., Tong, C., Zheng, J., 2015. Pressure decimation and interpolation (PDI) method for a baroclinic non-hydrostatic model. Oc. Mod. 96, 265-279. 
Skourup, J., Sterndorff, M.J., Hansen, E.A., 1992. Numerical modelling of wave-structure interaction by a three-dimensional non-linear boundary element method: A step towards the numerical wave tank. Oc. Engrg. 5, 437-460.

Smagorinsky, J., 1963. General circulation experiments with the primitive equations, i. the basic experiment. Monthly Weather Rev. 91, 99-164.

Soliman, M. S., Thompson, J. M. T., 1991. Transient and steady state analysis of capsize phenomena. Appl. Oc. Res. 13(2), 82-92.

Song, A., Morriss, B.F., Deeb, E.J., Richter-Menge, J., Perovich, D.K., Hopkins, M.A., 2014. High resolution sea ice dynamics modeling using the discrete element method. Amer. Geophys. Union Fall Mtg. 2014, Abstract C54A-08.

Soomere, Tarmo, 2005. Fast ferry traffic as a qualitatively new forcing factor of environmental processes in non-tidal sea areas: a case study in Tallinn Bay, Baltic Sea. Envir. Fl. Mech. 5, 293-323.

Soomere, T., 2007. Nonlinear components of ship wake waves. Appl. Mech. Rev. 60(1-6), 120-138.

Spyrou, K. J., Thompson, J. M. T., 2000. The nonlinear dynamics of ship motions: a field overview and some recent developments. Phil. Trans. R. Soc. Lond. A 358, 1735-1760.

Squire, V.A., Dugan, J.P., Wadhams, P., Rottier, P.J., Liu, A.K., 1995. Of ocean waves and sea ice. Annu. Rev. Fluid Mech. 27, 115-168.

Squire, V.A., 2007. Of ocean waves and sea-ice revisited. Cold Reg. Sci. and Tech. 49, 110-133.

Stelling, G., Zijlema, M., 2003. An accurate and efficient finite-difference algorithm for non-hydrostatic free surface flow with application to wave propagation. Intl. J. Numer. Methods Fluids 43, 1-23. 
Thomson, J., Squire, V., Rogers, E., Babanin, A., Guest, P., Maksym, T., Wadhams, P., Stammerjohn, S., Fairall, C., Persson, O., Doble, M., Graber, H., Shen, H., Gemmrich, J., Lehner, S., Holt, B., Williams, T., Meylan, M., Bidlot, J., 2013. Sea state and boundary layer physics of the emerging Arctic Ocean. Technical Report, APL-UW TR1306, Appl. Phys. Lab., U. of Washington, Seattle, 59pp.

Thomson, J., Rogers, W.E., 2014. Swell and sea in the emerging Arctic Ocean. Geophys. Res. Letters 41, 3136-3140.

Toffoli, A., Bennetts, L.G., Meylan, M.H., Cavaliere, C., Alberello, A., Elsnab, J., Monty, J.P., 2015. Sea ice floes dissipate the energy of steep ocean waves. Geophys. Res. Lett., 42, 8547-8554.

Tolman, H.L., 2009. User Manual and System Documentation of WAVEWATCH III(TM) Version 3.14. Tech. Note, NOAA/NWS/NCEP/MMAB, 220 pp..

Torsvik, T., Didenkulova, I., Soomere, T., 2009. Variability in spatial patterns of long nonlinear waves from fast ferries in Tallinn Bay. Nonlinear Proc. in Geophys. 16(2), 351-363.

Toyota, T., Takatsuji, S., Nakayama, M., 2006. Characteristics of sea ice floe size distribution in the seasonal ice zone. Geophys. Res. Ltrs. 33, L02616.

Wadhams, P., 1973. The effect of a sea ice cover on ocean surface waves. PhD thesis, Univ. Cambridge, UK, 223 pp.

Wadhams, P., 1986. The seasonal ice zone/The Geophysics of Sea Ice. Springer, USA, 825-991.

Wadhams, P., Squire, V.A., Goodman, D.J., Cowan, A.M., Moore, S.C., 1988. The attenuation rates of ocean waves in the marginal ice zone. J. Geophys. Res. 93(C6), 6799-6818.

Wall, W. A., Genkinger, S., Ramm, E., 2007. A strong coupling partitioned approach for fluid-structure interaction with free surfaces. Computers \& Fluids 36, 169-183. 
Wei, G., Kirby, J. T., Grilli, S. T., Subramanya, R., 1995. A fully nonlinear Boussinesq model for surface waves. Part 1. Highly nonlinear unsteady waves. J. Fluid Mech. 294, 71-92.

Williams, T.D., Bennetts, L.G., Dumont, D., Squire, V.A., Bertino, L., 2013. Wave-ice interactions in the marginal ice zone, Part 1: Theoretical foundations. Ocean Mod. 71, 81-91.

Xu, Z., Tartakovsky, A. M., Pan, W., 2012. Discrete-element model for the interaction between ocean waves and sea ice. Phys. Rev. E 85, 016703.

Yiew, L.J., Bennetts, L.G., Meylan, M.H., French, B.J., Thomas, G.A., 2016. Hydrodynamic responses of a thin floating disk to regular waves. Ocean Mod. $97,52-64$.

Zheng, Y. H., You, Y. G., Shen, Y. M., 2004. On the radiation and diffraction of water waves by a rectangular buoy. Ocean Engrg. 31, 1063-1082.

Zhao, X., Shen, H.H., 2015. Ocean wave transmission and reflection by viscoelastic ice covers. Ocean Mod. 92, 1-10.

Zhao, X., Shen, H.H., Cheng, S., 2015. Modeling ocean wave propagation under sea ice covers. Acta Mech. Sinica 31(1), 1-15.

Zhao, X., Shen, H.H., 2013. Ocean wave transmission and reflection between two connecting viscoelastic ice covers: An approximate solution. Ocean Mod. 71, 102-113. 


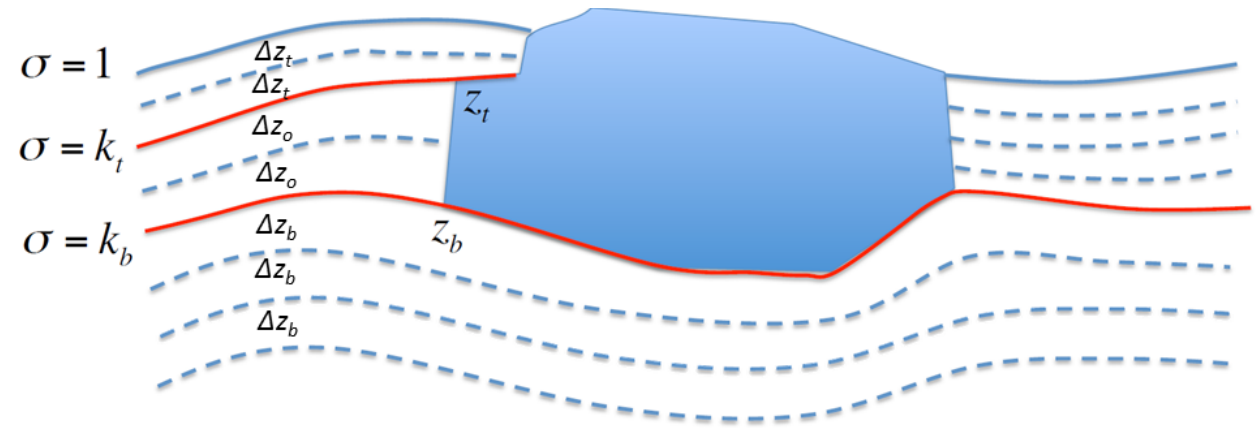

Figure 1: $\sigma$-coordinate transformation.

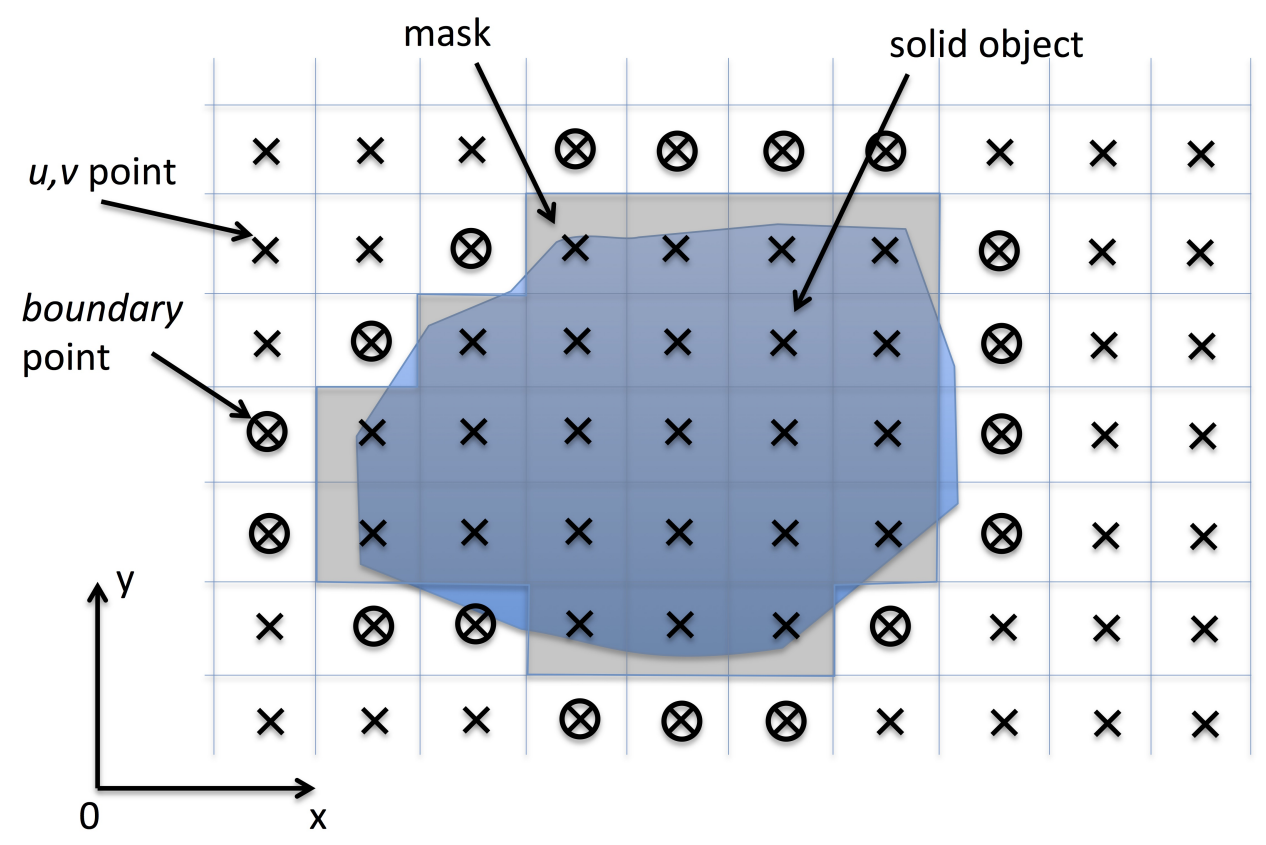

Figure 2: 3D masks (gray color) in the $\mathrm{x}-\mathrm{y}$ plane. Object is plotted in blue. Crosses: $u$ and $v$ points. Circled crosses: boundary points for the lateral immersed boundary conditions. 


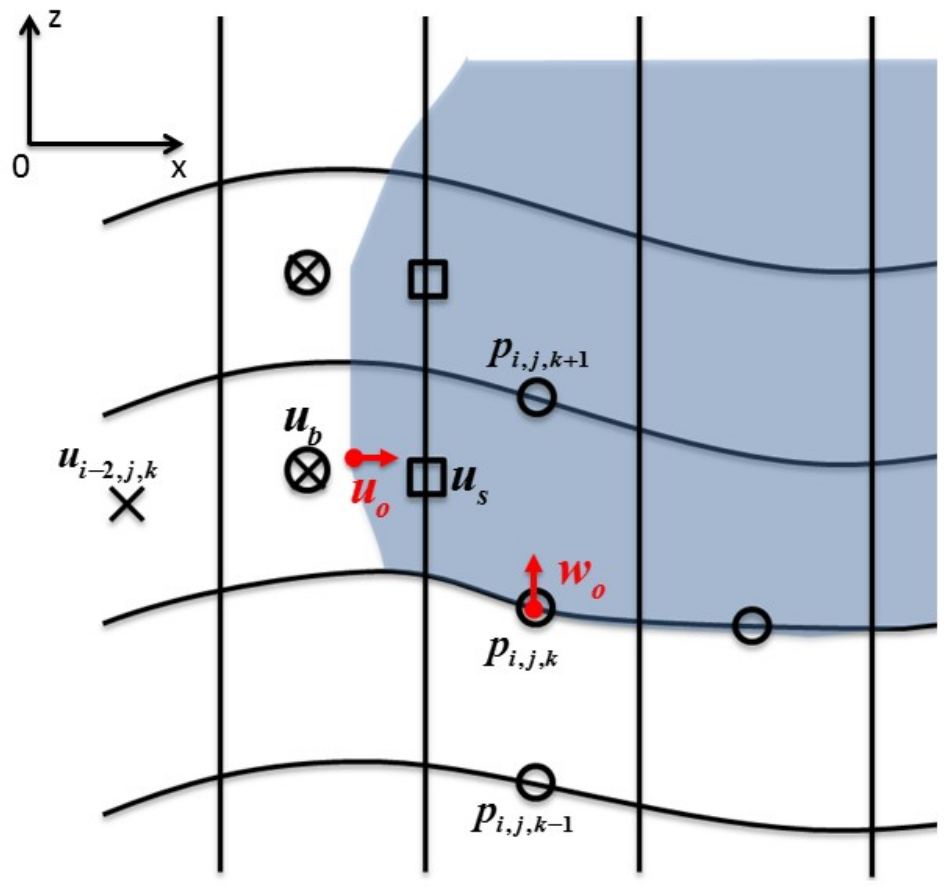

Figure 3: Sketch for the immersed boundary condition in the $\mathrm{x}-\mathrm{z}$ plane with pressure (circles), velocity (crosses), boundary points for the lateral immersed boundary condition (circled crosses), cell boundary fluxes (squares), and marker points for local object velocity (red circles/arrows). 


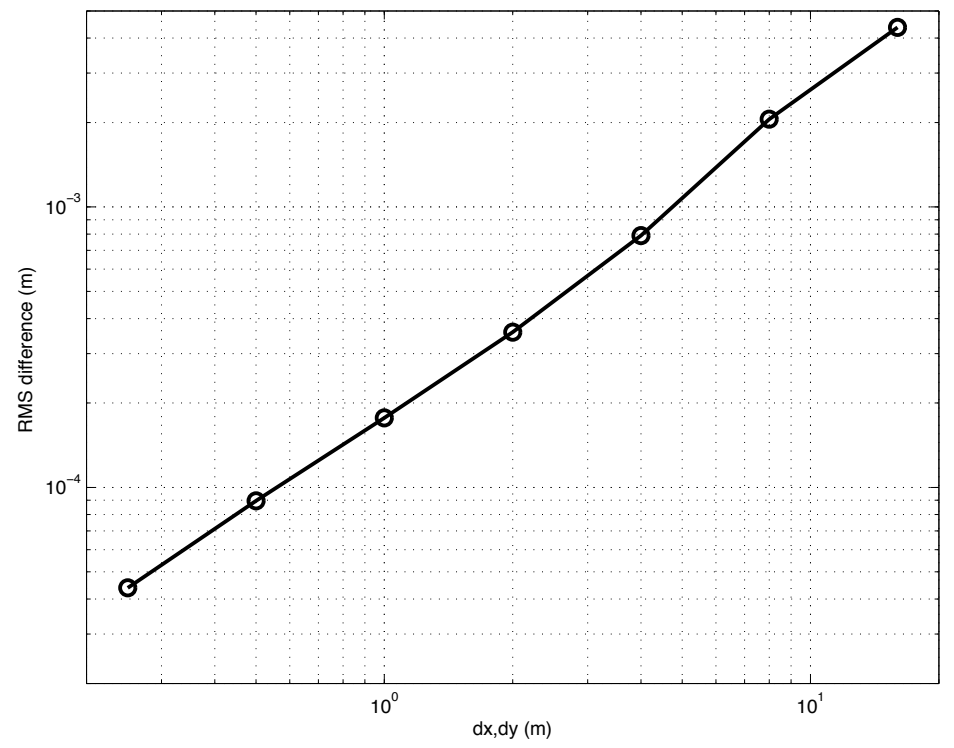

Figure 4: Convergence rates with horizontal grid refinement.

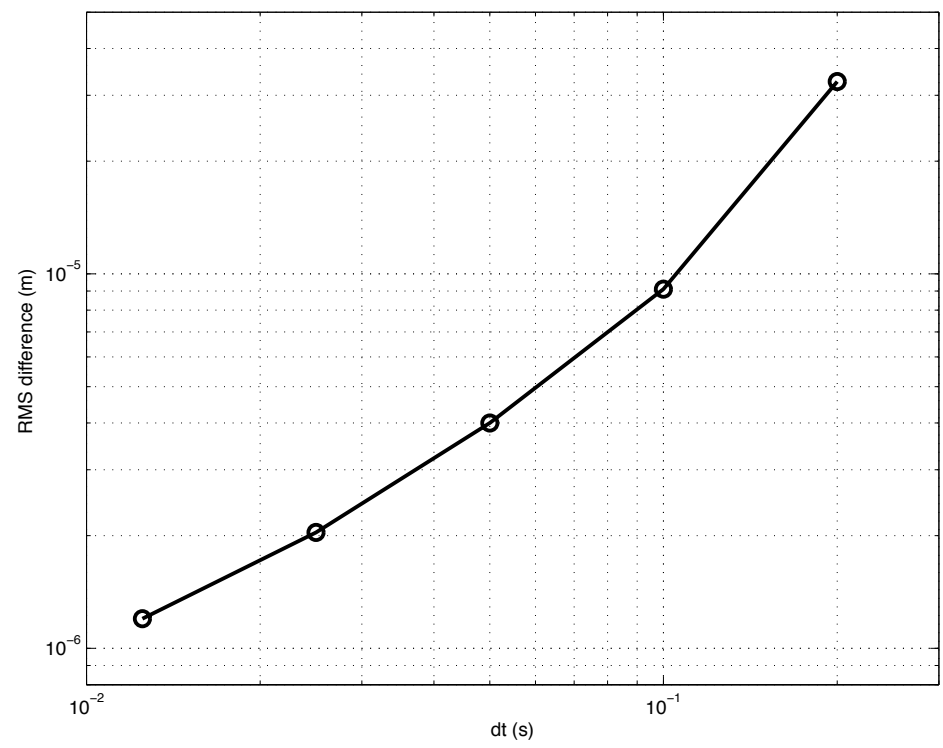

Figure 5: Convergence rates with time step refinement. 


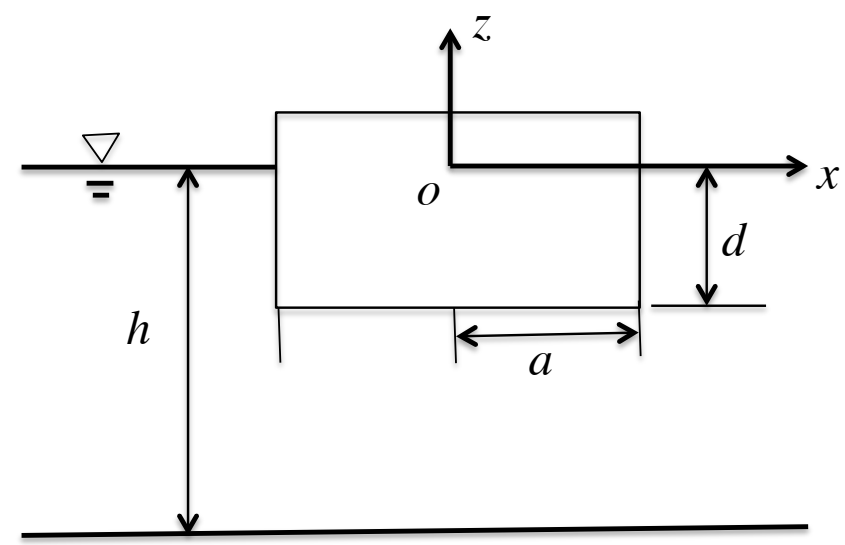

Figure 6: Sketch of 2D floating rectangular object. 


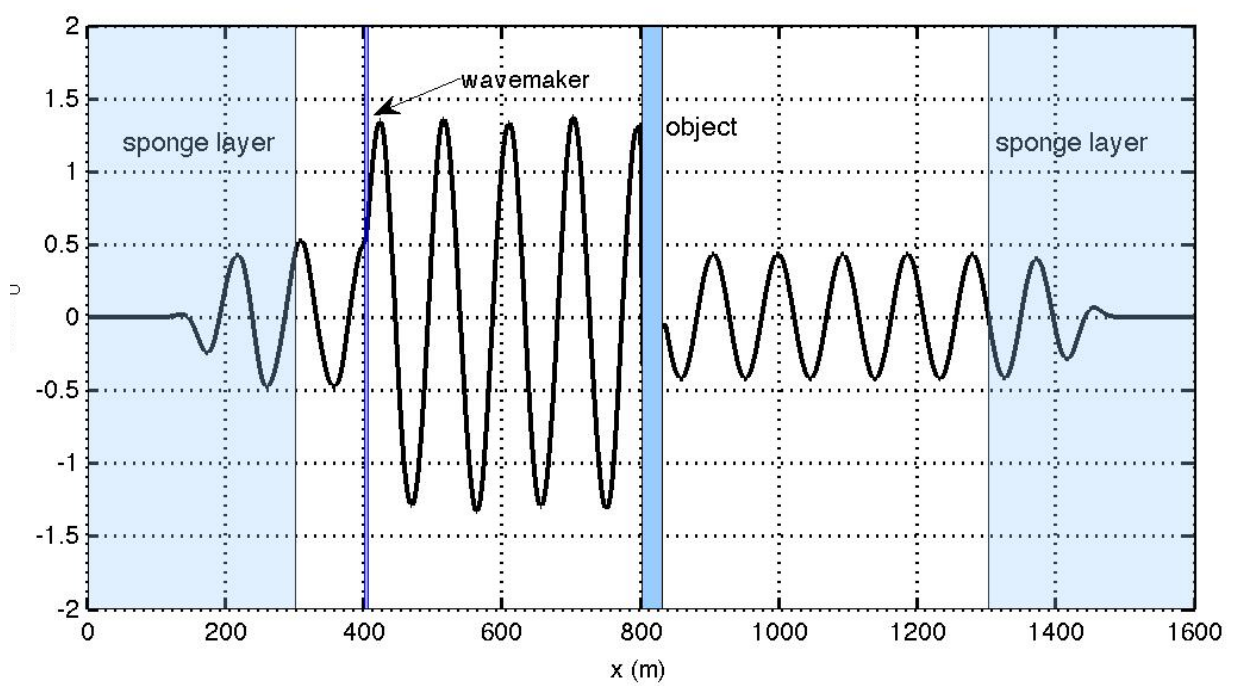

Figure 7: Snapshot of wave reflection and transmission by a floating object. 


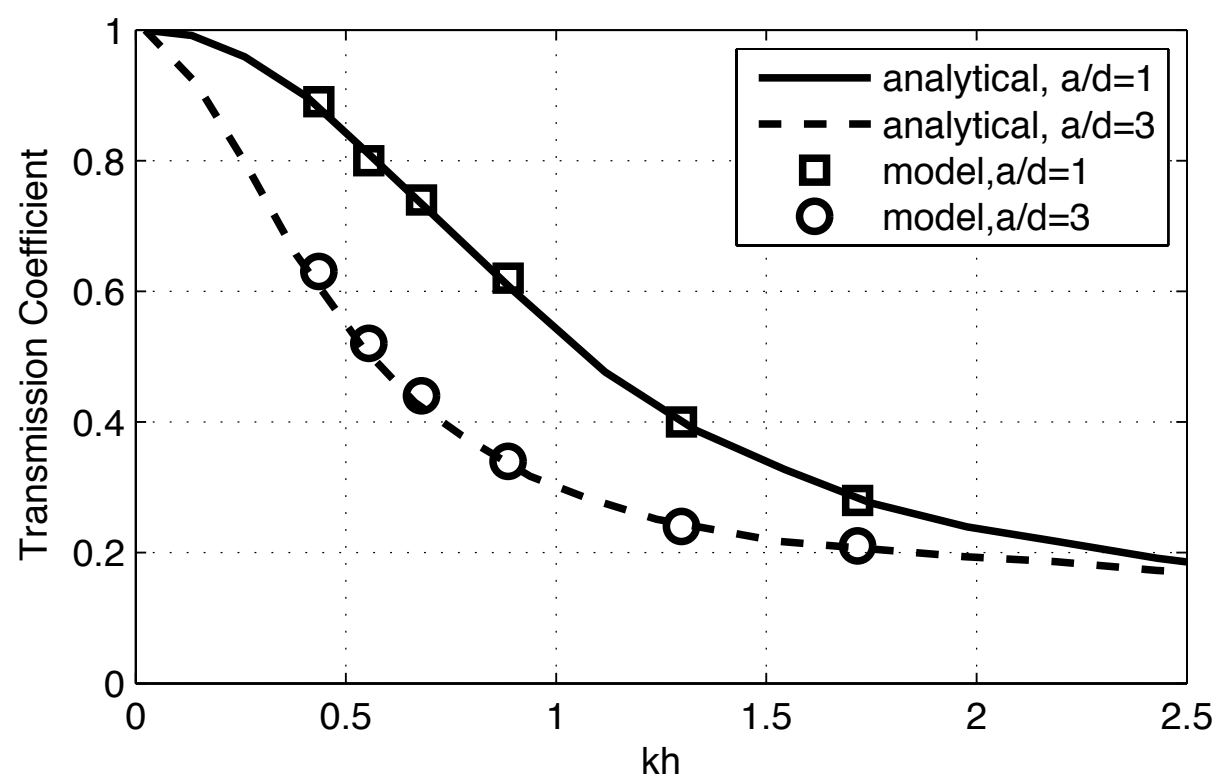

Figure 8: Comparisons between model results and analytical solution of wave transmission past a floating object. 


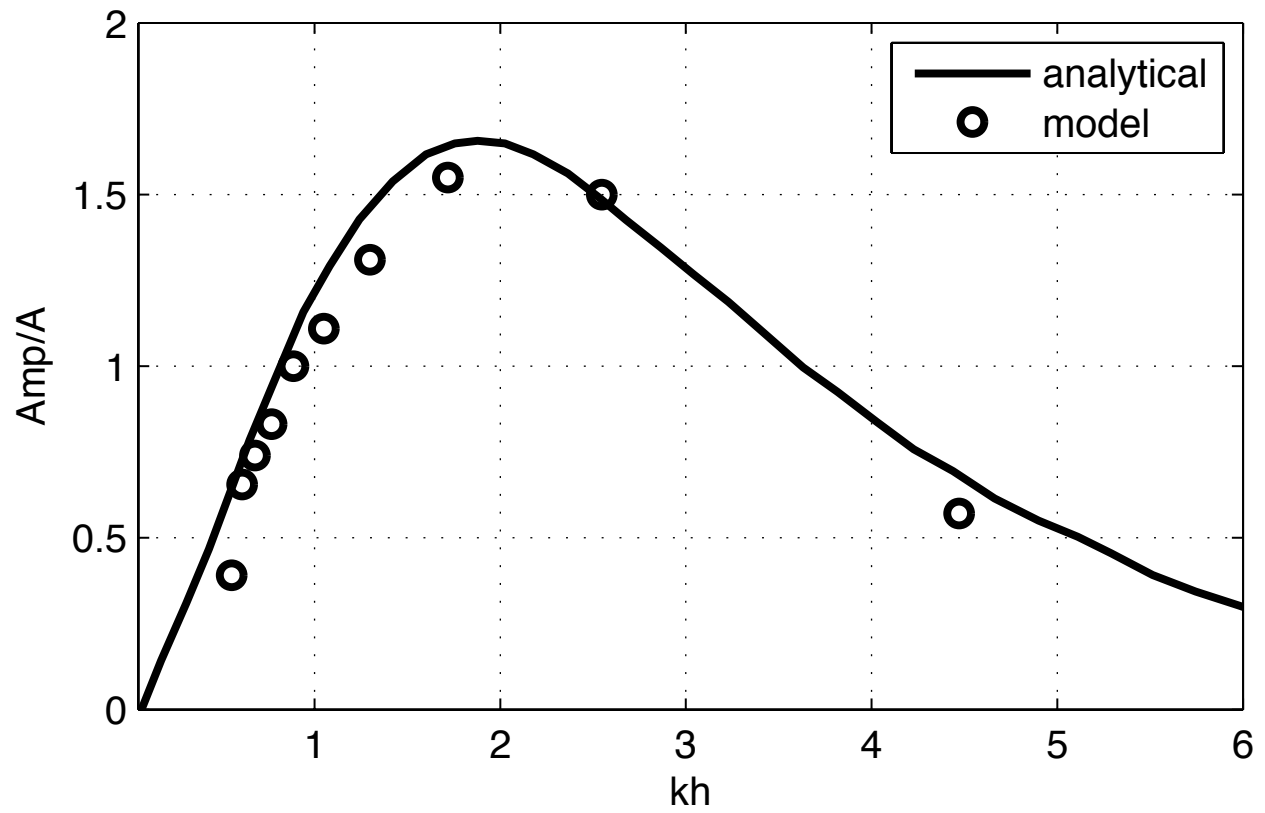

Figure 9: Comparisons between model results and analytical solution of waves generated by object heaving. 


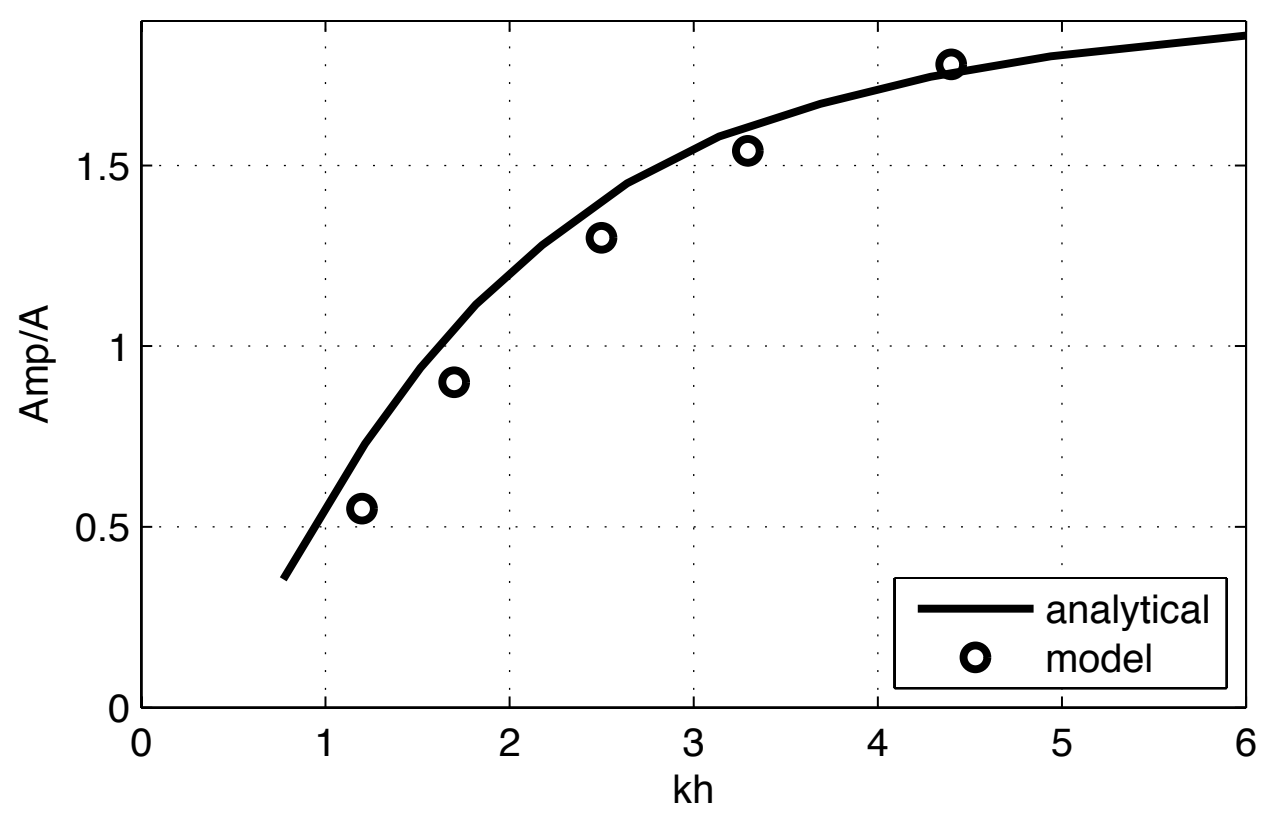

Figure 10: Comparisons between model results and analytical solution of waves generated by object surging. 


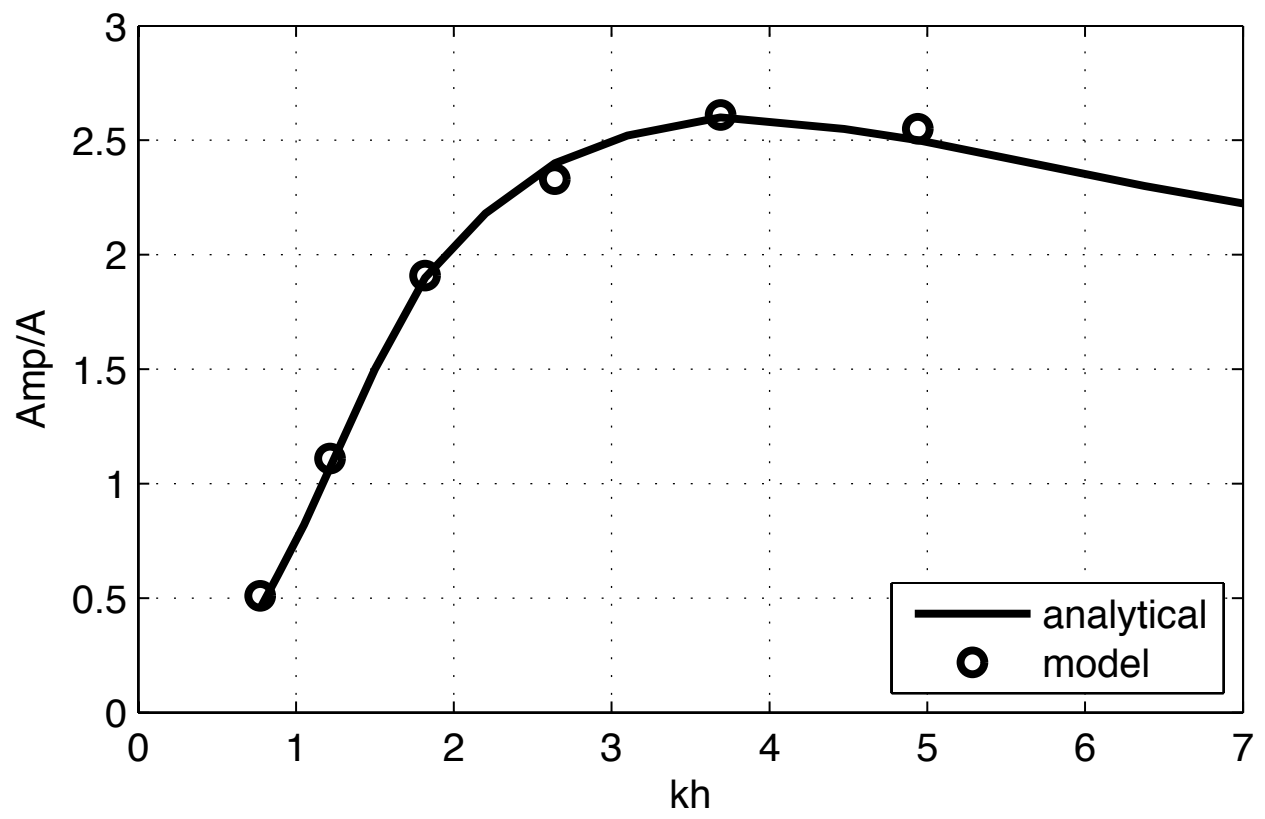

Figure 11: Comparisons between model results and analytical solution of waves generated by object pitching. 

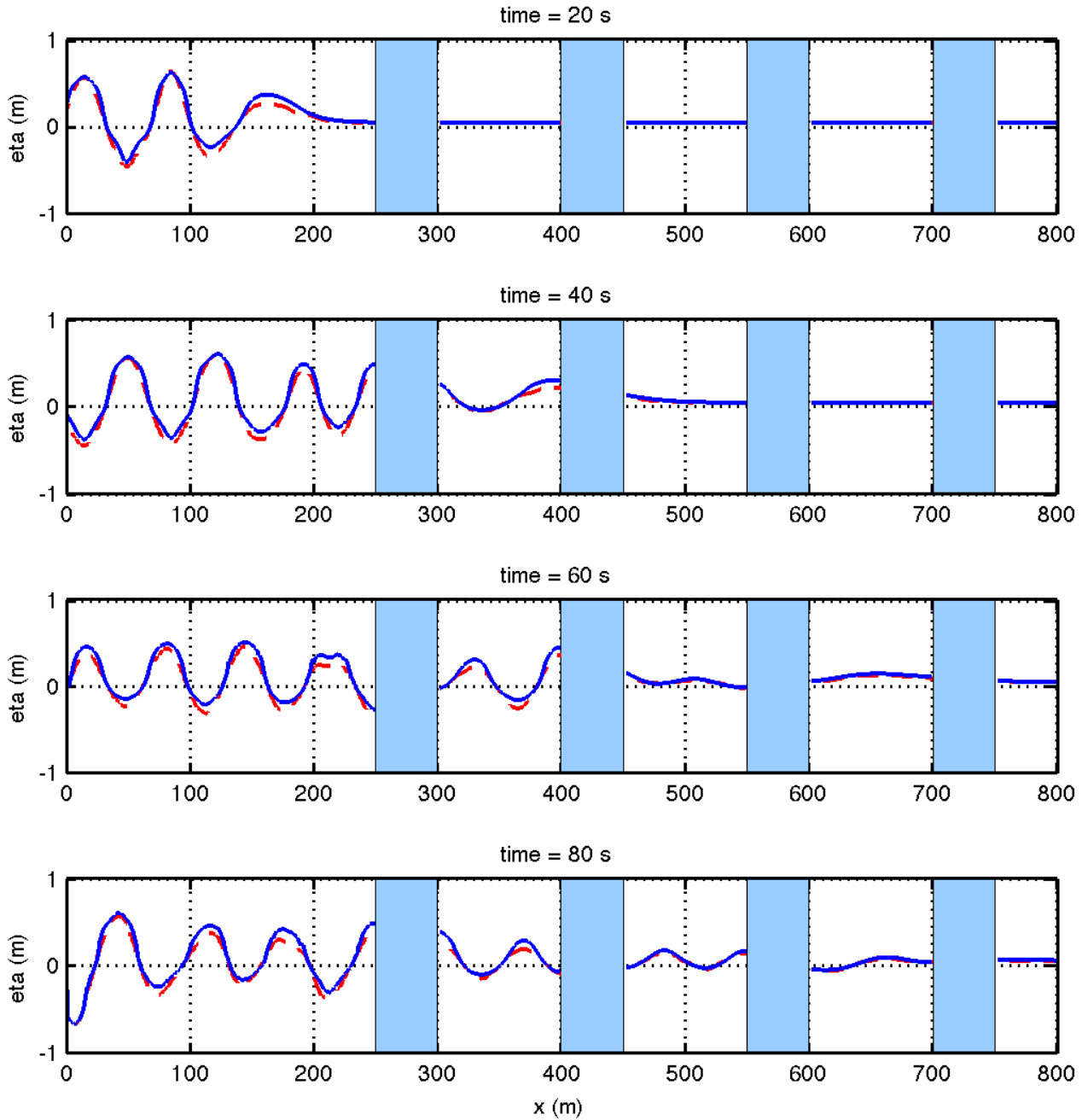

Figure 12: Comparisons between NHWAVE (blue solid lines) and VOF model (red dashed lines) showing transmission and reflection of steep wave components by floating objects (light blue rectangles). 


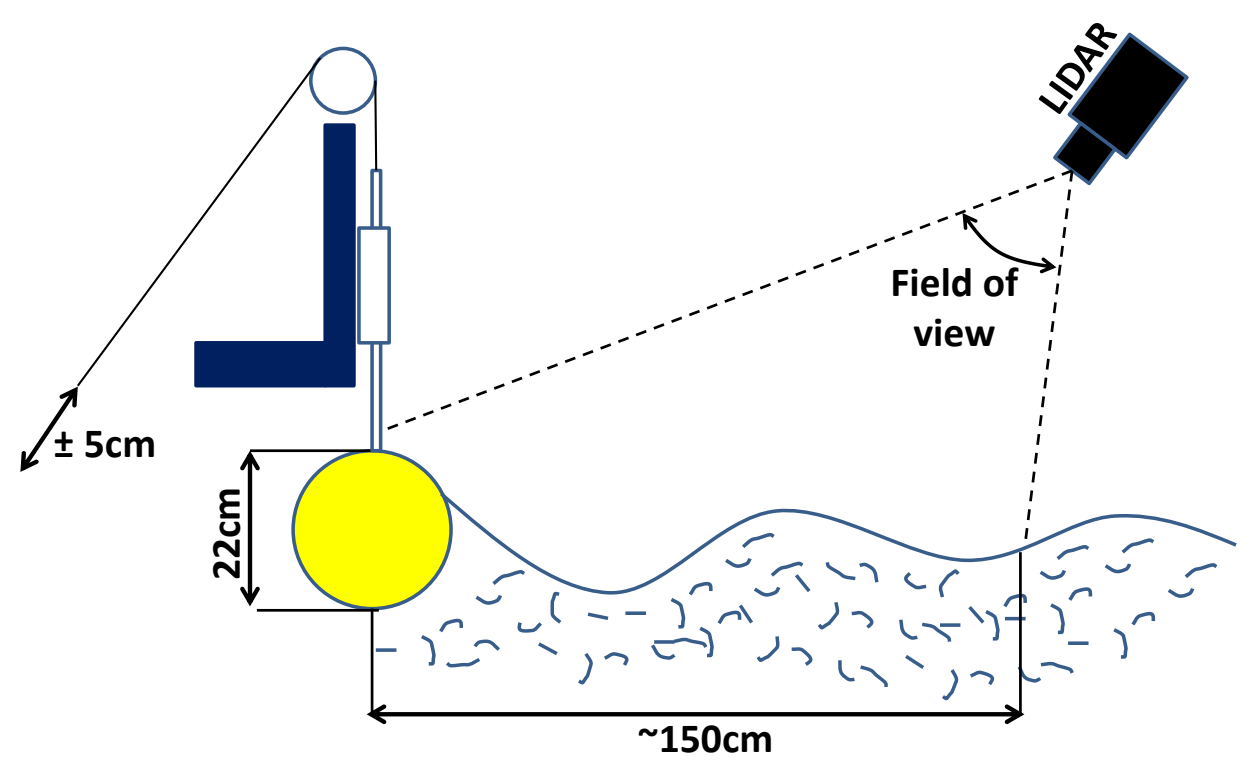

Figure 13: Experimental setup for wave generation by oscillating sphere, side view. 

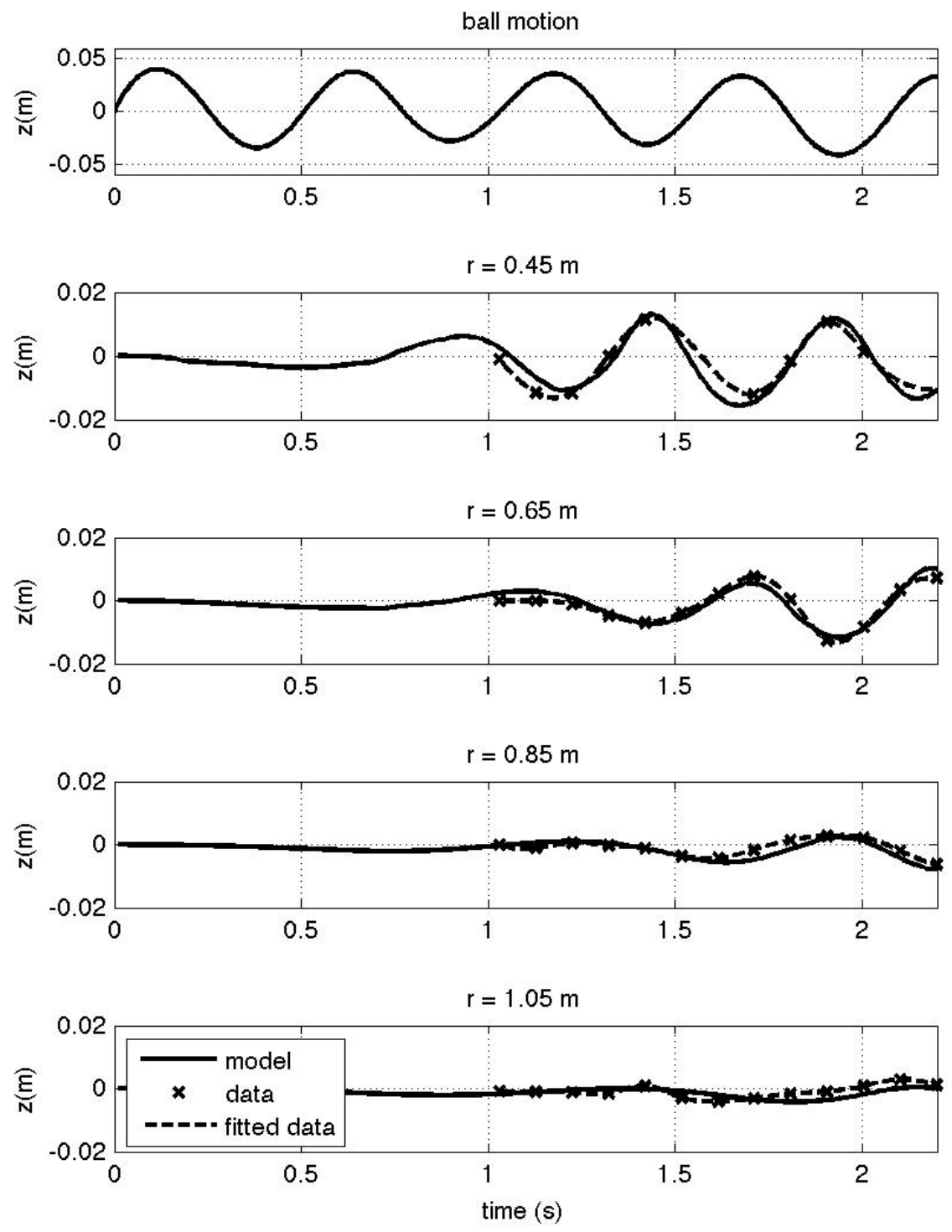

Figure 14: Elevation time series from oscillating sphere experiment, showing vertical position of sphere center (top panel) and water elevation at increasing distance $(r)$ from sphere center (remaining panels). Solid line is model, $x$ is LIDAR data, and dashed line is fitted to data. 


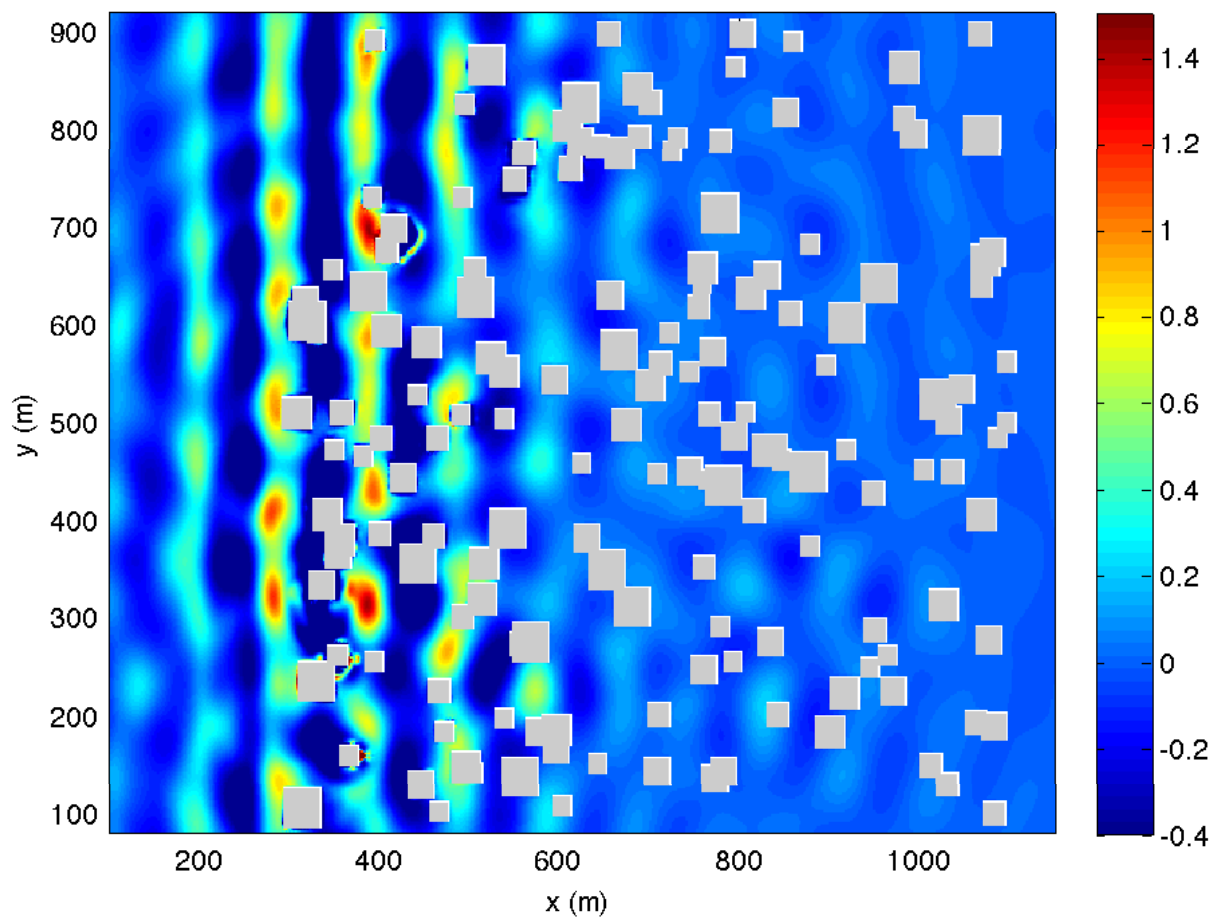

Figure 15: Snapshot of surface elevation in a simulation of irregular waves passing random distributed ice floes. Colorbar represents surface elevation. 